QUARTERLY OF APPLIED MATHEMATICS

VOLUME LXIV, NUMBER 1

MARCH 2006, PAGES 105-136

S 0033-569X(06)00987-0

Article electronically published on January 24, 2006

\title{
MIXED PROBLEM FOR THE LAPLACE EQUATION OUTSIDE CUTS IN A PLANE WITH SETTING DIRICHLET AND SKEW DERIVATIVE CONDITIONS ON DIFFERENT SIDES OF THE CUTS
}

\author{
BY \\ P. A. KRUTITSKII (Dept. of Mathematics, Faculty of Physics, Moscow State University, Moscow \\ 117234, Russia) \\ AND
}

A. I. SGIBNEV (Dept. of Mathematics, Faculty of Physics, Moscow State University, Moscow 117234, Russia)

\begin{abstract}
The mixed problem for the Laplace equation outside cuts in a plane is considered. The Dirichlet condition is posed on one side of each cut and the skew derivative condition is posed on the other side. This problem generalizes the mixed Dirichlet-Neumann problem. Integral representation for a solution of the boundary value problem is obtained in the form of potentials. The densities in the potentials satisfy the uniquely solvable Fredholm integral equation of the second kind and index zero. Uniqueness and existence theorems for a solution of the boundary value problem are proved. Singularities of the gradient of the solution of the boundary value problem at the tips of the cuts are studied. Asymptotic formulas for singularities are obtained. The effect of the disappearance of singularities is discussed.
\end{abstract}

Introduction. The skew derivative problem for the Laplace equation outside cuts in a plane describes electric current from electrodes in a semiconductor film [9]. The mixed problem for the Laplace equation outside cuts in a plane with setting Dirichlet and skew derivative conditions on different sides of the cuts arises in the physics of semiconductors as well [2]. Cuts model electrodes in a semiconductor film. The Dirichlet condition specifies the electric potential, while the skew derivative condition specifies the normal component of the electric current from an electrode in a magnetized semiconductor. The mixed problem for the Laplace equation in which the Dirichlet condition is posed on one set of cuts and the skew derivative condition is posed on the other set of cuts was investigated in [10. In the present paper, we study a mixed problem with the Dirichlet condition on one side of each cut and the skew derivative condition on the other side. We obtain integral representation for a solution of the boundary value problem in the form

Received March 10, 2005.

2000 Mathematics Subject Classification. Primary 35J05, 45E05.

This work was partly supported by the RFBR grant no. 05-01-00050.

(C)2006 Brown University 
of potentials for the cuts of an arbitrary shape. Substituting this integral representation in the form of potentials into the boundary conditions we derive the system of integral equations for the densities in potentials. The system involves Cauchy singular integral equations and additional conditions. Next this system for the densities in the potentials is reduced to the uniquely solvable Fredholm integral equation of the second kind and index zero in the appropriate Banach space. Uniqueness and existence theorems for a solution of the boundary value problem are proved. The integral representation for a solution of the boundary value problem is used to derive asymptotic formulas for singularities of a solution gradient at the tips of the cuts. The effect of the disappearance of singularities at the tips under certain conditions is discussed. The particular case of the present boundary value problem has been studied in [2, where cuts placed along a straight line have been considered. Another particular case of this problem has been treated in [1, where Dirichlet and Neumann conditions were specified on different sides of the cuts of an arbitrary shape.

1. Statement of the problem. In a plane $x=\left(x_{1}, x_{2}\right) \in R^{2}$, we consider a set of simple open $\operatorname{arcs} \Gamma_{1}, \ldots, \Gamma_{N}$ of class $C^{2, \lambda}, \lambda \in(0,1]$. The arcs are assumed to be disjoint (including the endpoints). This set of arcs is referred to as the contour $\Gamma$. Let the contour $\Gamma$ be parametrized by the arc length $s$ :

$$
\Gamma_{n}=\left\{x: x=x(s)=\left(x_{1}(s), x_{2}(s)\right), s \in\left[a_{n}, b_{n}\right]\right\}, \quad n=1,2, \ldots, N .
$$

We choose the parametrization in such a way that for different $n$, the closed intervals $\left[a_{n}, b_{n}\right]$ are disjoint including the endpoints. The set of closed intervals $O s$ corresponding to the contour $\Gamma$ is also denoted by $\Gamma$. The tangent vector to $\Gamma$ at the point $x(s)$ is denoted by $\tau_{x}=(\cos \alpha(s), \sin \alpha(s))$, and the normal is denoted by $\mathbf{n}_{x}=(\sin \alpha(s),-\cos \alpha(s))$, i.e., $\cos \alpha(s)=x_{1}^{\prime}(s), \sin \alpha(s)=x_{2}^{\prime}(s)$. Let a plane be cut along the contour $\Gamma$. By $\Gamma^{+}$we denote the left side of $\Gamma$ as $s$ increases, and by $\Gamma^{-}$we denote the opposite side. By the indices + and - we denote the limit values of functions on $\Gamma^{+}$and $\Gamma^{-}$, respectively.

We say that a function $u(x)$ belongs to the class $\mathbf{G}$ if:

1) $u(x) \in C^{0}\left(\overline{R^{2} \backslash \Gamma}\right)$ and $u(x)$ is continuous at the endpoints of $\Gamma$;

2) $\nabla u(x) \in C^{0}\left(\overline{R^{2} \backslash \Gamma} \backslash X\right)$, where $X=\bigcup_{n=1}^{N}\left(x\left(a_{n}\right) \cup x\left(b_{n}\right)\right)$ is the set of endpoints of $\Gamma$;

3) the relation

$$
|\nabla u| \leq \text { const }|x-x(d)|^{\delta}
$$

is valid as $x \rightarrow x(d) \in X$, where const $>0, \delta>-1$, and either $d=a_{n}$ or $d=b_{n}$ $(n=1, \ldots, N)$.

By $C^{0}\left(\overline{R^{2} \backslash \Gamma}\right)$ we denote the class of functions that have left and right limit values on the cut $\Gamma$; these values can be different in the inner points of $\Gamma$, i.e., functions may have a jump on $\Gamma$.

Let us state the mixed skew derivative problem for the Laplace equation outside the set of cuts in a plane.

Problem $\mathcal{S}$. To find a function $u(x) \in \mathbf{G}$ that satisfies the Laplace equation

$$
\Delta u(x)=0, \quad x \in R^{2} \backslash \Gamma,
$$


the boundary conditions

$$
\begin{gathered}
\left.u(x)\right|_{x(s) \in \Gamma^{+}}=f^{+}(s), \\
\left.\left(\frac{\partial u}{\partial \mathbf{n}_{x}}+\beta \frac{\partial u}{\partial \tau_{x}}\right)\right|_{x(s) \in \Gamma^{-}}=f^{-}(s),
\end{gathered}
$$

and the conditions at infinity

$$
u(x)=A \ln |x|+O(1), \quad \frac{\partial u}{\partial|x|}=\frac{A}{|x|}+O\left(|x|^{-2}\right), \quad|x| \rightarrow \infty .
$$

We assume that $f^{+}(s), f^{-}(s)$ are given functions; $A$ and $\beta$ are given constants. For $A=0$, we have the classical boundedness condition at infinity. For $\beta=0$, we have the mixed Dirichlet-Neumann problem [1].

Let us prove the uniqueness theorem.

Theorem 1. Problem $\mathcal{S}$ has at most one solution.

Proof. Suppose that Problem $\mathcal{S}$ has two solutions $u_{1}(x), u_{2}(x) \in \mathbf{G}$. Then the function $u_{0}(x)=u_{1}(x)-u_{2}(x)$ belongs to the class $\mathbf{G}$ and satisfies Problem $\mathcal{S}$ with the homogeneous boundary conditions (3) and (4) and with the conditions

$$
u_{0}(x)=O(1), \quad \frac{\partial u_{0}}{\partial|x|}=O\left(|x|^{-2}\right), \quad|x| \rightarrow \infty
$$

at infinity. By $l_{r}$ we denote the circumference of large radius $r$ centered at the origin. We surround each cut $\Gamma_{n}, n=1, \ldots, N$, by a closed curve. In the domain bounded with this closed curves and the circle $l_{r}$, we write out the energy identity for Eq. (2). We shrink closed curves to the cuts $\Gamma$, let $r \rightarrow \infty$ and use the smoothness properties of the function $u_{0}(x)$ and condition (6). Then the energy identity acquires the form

$$
\left\|\nabla u_{0}\right\|_{L_{2}\left(R^{2} \backslash \Gamma\right)}^{2}=\lim _{r \rightarrow \infty}\left\|\nabla u_{0}\right\|_{L_{2}\left(C_{r} \backslash \Gamma\right)}^{2}=\int_{\Gamma} u_{0}^{+}\left(\frac{\partial u_{0}}{\partial \mathbf{n}_{x}}\right)^{+} d s-\int_{\Gamma} u_{0}^{-}\left(\frac{\partial u_{0}}{\partial \mathbf{n}_{x}}\right)^{-} d s,
$$

where $C_{r}$ is the circle of radius $r$ centered at the origin. The first integral equals zero by virtue of the homogeneous boundary condition (3). It follows from the homogeneous condition (3) and from the definition of class $\mathbf{G}$ that $u_{0}\left(a_{n}\right)=u_{0}\left(b_{n}\right)=0, n=1, \ldots, N$. Putting $\left(\partial u_{0} / \partial \mathbf{n}_{x}\right)^{-}=-\beta\left(\partial u_{0} / \partial \tau_{x}\right)^{-}$to the second integral we obtain

$$
\left\|\nabla u_{0}\right\|_{L_{2}\left(R^{2} \backslash \Gamma\right)}^{2}=\beta \int_{\Gamma} u_{0}^{-}\left(\frac{\partial u_{0}}{\partial \tau_{x}}\right)^{-} d s=\frac{\beta}{2} \sum_{n=1}^{N}\left(u_{0}^{2}\left(b_{n}\right)-u_{0}^{2}\left(a_{n}\right)\right)=0 .
$$

Hence $u_{0}(x) \equiv$ const in $R^{2} \backslash \Gamma$. The homogeneous condition (3) gives us const $=0$. Therefore, $u_{0}(x) \equiv 0$. The proof is complete.

2. Reduction to a system of singular integral equations. To prove the existence theorem, we impose additional smoothness conditions on the functions occurring in the boundary conditions (3) and (4); namely,

$$
f^{+}(s) \in C^{1, \lambda}(\Gamma), \quad f^{-}(s) \in C^{0, \lambda}(\Gamma), \quad \lambda \in(0,1] .
$$


Note that the Hölder coefficient $\lambda$ in the smoothness conditions for the contour $\Gamma$ and that for the functions $f^{+}(s)$ and $f^{-}(s)$ are assumed to be the same. If these coefficients are different, then as $\lambda$ we can take the smaller of them.

We replace condition (3) by the following equivalent boundary conditions on $\Gamma$ :

$$
\begin{gathered}
\left.\frac{\partial u}{\partial \tau_{x}}\right|_{x(s) \in \Gamma^{+}}=f^{\prime}+(s), \quad f^{\prime}(s) \equiv \frac{d f^{+}(s)}{d s} \in C^{0, \lambda}(\Gamma), \\
u\left(x\left(a_{n}\right)\right)=f^{+}\left(a_{n}\right), \quad n=1, \ldots, N .
\end{gathered}
$$

Here we have taken into account that the relation $\partial / \partial \tau_{x}=\partial / \partial s$ is valid at each point $x(s) \in \Gamma$ by virtue of chosen parametrization.

We seek a solution in the form

$$
u[\mu, \nu](x)=V[\mu](x)+T[\nu](x)+B_{2 N},
$$

where $B_{2 N}$ is a real constant (the notation $B_{2 N}$ is clarified below),

$$
V[\mu](x)=-\frac{1}{2 \pi} \int_{\Gamma} \mu(s) \ln |x-y(s)| d s
$$

is a single layer potential for the Laplace equation (2),

$$
T[\nu](x)=-\frac{1}{2 \pi} \int_{\Gamma} \nu(s) \psi(x, y(s)) d s
$$

is an angular potential [3, 4, for the Laplace equation (2).

The unknown functions $\mu(s), \nu(s)$ defined on $\Gamma$ will be looked for in the space $C_{q}^{\varpi}(\Gamma)$, $\varpi \in(0,1], q \in[0,1)$.

We say that a function $\mathcal{F}(s)$ defined on $\Gamma$ belongs to the Banach space $C_{q}^{\varpi}(\Gamma), \varpi \in$ $(0,1], q \in[0,1)$, if

$$
\mathcal{F}_{0}(s)=\mathcal{F}(s) \prod_{n=1}^{N}\left|s-a_{n}\right|^{q}\left|s-b_{n}\right|^{q} \in C^{0, \varpi}(\Gamma) .
$$

The norm in the space $C_{q}^{\varpi}(\Gamma)$ is given by the relation

$$
\left\|\left.\mathcal{F}(s)\right|_{C_{q}^{\varpi}(\Gamma)}=\right\| \mathcal{F}_{0}(s) \|_{C^{0, \varpi}(\Gamma)} .
$$

The kernel of the angular potential is defined up to $2 \pi m$ (where $m$ is an integer) by the formulas

$$
\cos \psi(x, y)=\frac{x_{1}-y_{1}}{|x-y|}, \quad \sin \psi(x, y)=\frac{x_{2}-y_{2}}{|x-y|} .
$$

Obviously, $\psi(x, y)$ is the angle between the vector $\overrightarrow{y x}$ and the direction of the axis $O x_{1}$. On the other hand, $\psi(x, y)$ is the multivalued harmonic function conjugate to $\ln |x-y|$ in the sense of the Cauchy-Riemann relations.

Let $x$ be an arbitrary given point of a plane lying outside $\Gamma$, and let $y=y(s) \in \Gamma$. Then $\psi(x, y(s))$ is treated as an arbitrary fixed branch of this function, which is continuous with respect to $s$ along $\Gamma$. In view of this definition of $\psi(x, y(s))$, the angular potential 
is a multivalued function. The angular potential is single valued under the following conditions [3, 4]:

$$
\int_{\Gamma_{n}} \nu(s) d s=0, \quad n=1, \ldots, N .
$$

Under these conditions, the harmonic angular potential can be represented as the harmonic double-layer potential

$$
T[\nu](x)=\frac{1}{2 \pi} \int_{\Gamma} w(s) \frac{\partial \ln |x-y(s)|}{\partial \mathbf{n}_{y}} d s,
$$

where

$$
w(s)=\int_{a_{n}}^{s} \nu(\sigma) d \sigma, \quad s \in\left[a_{n}, b_{n}\right], \quad n=1, \ldots, N .
$$

It follows from (11) that $w\left(a_{n}\right)=w\left(b_{n}\right)=0$ for $n=1, \ldots, N$.

If we require that

$$
\int_{\Gamma} \mu(s) d s=-2 \pi A,
$$

then the function $u[\mu, \nu](x)$ occurring in (10) satisfies condition (5) at infinity.

If the densities $\mu(s)$ and $\nu(s)$ belong to $C_{q}^{\varpi}(\Gamma), \varpi \in(0,1], q \in[0,1)$, and satisfy conditions (11), (12), then the function $u[\mu, \nu](x)$ in (10) belongs to the class $\mathbf{G}$ and satisfies all the conditions of Problem $\mathcal{S}$ except for the boundary conditions [3, 4, 5]. In particular, condition (1) is valid with $\delta=-q$ if $q \in(0,1)$.

Let us satisfy the conditions (4) and (8). Substituting the function (10) into these conditions and taking into account the limit formulas for the tangent and normal derivatives of the logarithmic and angular potentials [4, Theorem 5], we obtain the following system of two singular integral equations for $\nu(s)$ and $\mu(s)$ :

$$
\begin{aligned}
\frac{\nu(s)}{2}+ & \frac{1}{2 \pi} \int_{\Gamma} \nu(\sigma) \frac{\cos \phi_{0}(x(s), y(\sigma))}{|x(s)-y(\sigma)|} d \sigma+\frac{1}{2 \pi} \int_{\Gamma} \mu(\sigma) \frac{\sin \phi_{0}(x(s), y(\sigma))}{|x(s)-y(\sigma)|} d \sigma \\
& =f^{\prime}+(s), \quad s \in \Gamma, \\
& -\frac{\mu(s)+\beta \nu(s)}{2}+\frac{1}{2 \pi} \int_{\Gamma}(\mu(\sigma)+\beta \nu(\sigma)) \frac{\cos \phi_{0}(x(s), y(\sigma))}{|x(s)-y(\sigma)|} d \sigma \\
& -\frac{1}{2 \pi} \int_{\Gamma}(\nu(\sigma)-\beta \mu(\sigma)) \frac{\sin \phi_{0}(x(s), y(\sigma))}{|x(s)-y(\sigma)|} d \sigma=f^{-}(s), \quad s \in \Gamma .
\end{aligned}
$$

The second integrals in these equations are treated in the sense of the principal value. By $\phi_{0}(x(s), y(\sigma))$ we denote the angle between the vector $\overrightarrow{x y}$ and the direction of the normal $\mathbf{n}_{x}$ at the point $x \in \Gamma$. The angle is considered to be positive if it is measured anticlockwise from $\mathbf{n}_{x}$, and negative if it is measured clockwise from $\mathbf{n}_{x}$. Moreover, if $x \neq y$, then the angle $\phi_{0}(x, y)$ is assumed to be continuous with respect to $x$ and $y$ on $\Gamma$. The angles $\phi_{0}(x(s), y(\sigma))$ and $\psi(x(s), y(\sigma))$ are connected up to $2 \pi m$ (with integer $m$ ) by the formula $\phi_{0}(x(s), y(\sigma))=\psi(x(s), y(\sigma))-\alpha(s)-\pi / 2$. 
We introduce the notation

$$
Y_{2}(s, \sigma)=\frac{1}{\pi}\left[\frac{\sin \phi_{0}(x(s), y(\sigma))}{|x(s)-y(\sigma)|}-\frac{1}{\sigma-s}\right], \quad Y_{1}(s, \sigma)=\frac{1}{\pi} \frac{\cos \phi_{0}(x(s), y(\sigma))}{|x(s)-y(\sigma)|} .
$$

It was shown in [4, Lemmas 2(1) and 3(1)] that if $\Gamma \in C^{2, \lambda}$, then the functions $Y_{1}(s, \sigma)$ and $Y_{2}(s, \sigma)$ satisfy the Hölder condition with the exponent $\lambda$ with respect to both variables on $\Gamma$. We rewrite singular integral equations for $\nu(s)$ and $\mu(s)$ as follows:

$$
\begin{gathered}
\nu(s)+\frac{1}{\pi} \int_{\Gamma} \frac{\mu(\sigma)}{\sigma-s} d \sigma+\int_{\Gamma} \mu(\sigma) Y_{2}(s, \sigma) d \sigma+\int_{\Gamma} \nu(\sigma) Y_{1}(s, \sigma) d \sigma=2 f^{\prime}+(s), \quad s \in \Gamma, \\
\mu(s)+\beta \nu(s)+\frac{1}{\pi} \int_{\Gamma} \frac{\nu(\sigma)-\beta \mu(\sigma)}{\sigma-s} d \sigma+\int_{\Gamma}(\nu(\sigma)-\beta \mu(\sigma)) Y_{2}(s, \sigma) d \sigma \\
-\int_{\Gamma}(\mu(\sigma)+\beta \nu(\sigma)) Y_{1}(s, \sigma) d \sigma=-2 f^{-}(s), \quad s \in \Gamma .
\end{gathered}
$$

Putting the function (10) into conditions (9), we obtain the following additional conditions for $\mu(s), \nu(s)$, and $B_{2 N}$ :

$$
V[\mu]\left(x\left(a_{n}\right)\right)+T[\nu]\left(x\left(a_{n}\right)\right)+B_{2 N}=f^{+}\left(a_{n}\right), \quad n=1, \ldots, N .
$$

We have thereby obtained the system of integral equations (11)-(15) for the functions $\mu(s)$ and $\nu(s), s \in \Gamma$, and for the constant $B_{2 N}$. The preceding considerations imply the following assertion.

Theorem 2. If condition (7) is satisfied and system (11) (15) has a solution $\mu(s), \nu(s)$, $B_{2 N}$ such that $\mu(s), \nu(s) \in C_{q}^{\varpi}(\Gamma), \varpi \in(0,1], q \in[0,1)$, then the solution of Problem $\mathcal{S}$ exists and is given by (10).

Further on we analyze the solvability of the system of integral equations (11)-(15).

We perform a change of the unknown functions by the formulas

$$
\begin{gathered}
\rho_{1}(s)=\frac{\nu(s)-\left(\sqrt{1+\beta^{2}}+\beta\right) \mu(s)}{\left(\sqrt{1+\beta^{2}}+\beta\right)} \in C_{q}^{\varpi}(\Gamma), \\
\rho_{2}(s)=\frac{\mu(s)+\left(\sqrt{1+\beta^{2}}+\beta\right) \nu(s)}{\left(\sqrt{1+\beta^{2}}+\beta\right)} \in C_{q}^{\varpi}(\Gamma), \\
\nu(s)=\frac{\rho_{1}(s)+\left(\sqrt{1+\beta^{2}}+\beta\right) \rho_{2}(s)}{2 \sqrt{1+\beta^{2}}}, \\
\mu(s)=\frac{\rho_{2}(s)-\left(\sqrt{1+\beta^{2}}+\beta\right) \rho_{1}(s)}{2 \sqrt{1+\beta^{2}}} .
\end{gathered}
$$


In terms of $\rho_{1}(s), \rho_{2}(s)$, Eqs. (13) and (14) acquire the form

$$
\begin{aligned}
& \rho_{1}(s)-\left(\sqrt{1+\beta^{2}}+\beta\right) \frac{1}{\pi} \int_{\Gamma} \frac{\rho_{1}(\sigma)}{\sigma-s} d \sigma=F_{1}(s), \quad s \in \Gamma, \\
& \rho_{2}(s)+\left(\sqrt{1+\beta^{2}}-\beta\right) \frac{1}{\pi} \int_{\Gamma} \frac{\rho_{2}(\sigma)}{\sigma-s} d \sigma=F_{2}(s), \quad s \in \Gamma,
\end{aligned}
$$

where the functions

$$
\begin{gathered}
F_{1}(s)=2 \sqrt{1+\beta^{2}} f^{\prime}+(s)+2 f^{-}(s) \\
+\left(\sqrt{1+\beta^{2}}+\beta\right)\left(-\int_{\Gamma} \rho_{2}(\sigma) Y_{1}(s, \sigma) d \sigma+\int_{\Gamma} \rho_{1}(\sigma) Y_{2}(s, \sigma) d \sigma\right), \quad s \in \Gamma, \\
F_{2}(s)=2\left(\sqrt{1+\beta^{2}}-\beta\right)\left(\sqrt{1+\beta^{2}} f^{\prime}+(s)-f^{-}(s)\right) \\
-\left(\sqrt{1+\beta^{2}}-\beta\right)\left(\int_{\Gamma} \rho_{1}(\sigma) Y_{1}(s, \sigma) d \sigma+\int_{\Gamma} \rho_{2}(\sigma) Y_{2}(s, \sigma) d \sigma\right), \quad s \in \Gamma,
\end{gathered}
$$

belong to $C^{0, \lambda}(\Gamma)$.

In terms of $\rho_{1}(s), \rho_{2}(s)$ Eqs. (11), (12), and (15) acquire the form

$$
\begin{gathered}
\int_{\Gamma_{n}}\left(\rho_{1}(s)+\left(\sqrt{1+\beta^{2}}+\beta\right) \rho_{2}(s)\right) d s=0, \quad n=1, \ldots, N, \\
\int_{\Gamma}\left(\rho_{2}(s)-\left(\sqrt{1+\beta^{2}}+\beta\right) \rho_{1}(s)\right) d s=-4 \pi A \sqrt{1+\beta^{2}}, \\
V\left[\rho_{2}-\left(\sqrt{1+\beta^{2}}+\beta\right) \rho_{1}\right]\left(x\left(a_{n}\right)\right)+T\left[\rho_{1}+\left(\sqrt{1+\beta^{2}}+\beta\right) \rho_{2}\right]\left(x\left(a_{n}\right)\right) \\
+2 \sqrt{1+\beta^{2}} B_{2 N}=2 \sqrt{1+\beta^{2}} f^{+}\left(a_{n}\right), \quad n=1, \ldots, N .
\end{gathered}
$$

The system of integral equations for $\mu(s), \nu(s)$, and $B_{2 N}$ is thereby reduced to a system of equations for the functions $\rho_{1}(s), \rho_{2}(s)$, and $B_{2 N}$.

The main advantage of the resulting system is that the principle part of each equation (18), (19) contains only one unknown function (either $\rho_{1}(s)$ or $\rho_{2}(s)$ ).

Lemma 1. There is a one-to-one correspondence between solutions $\mu(s), \nu(s) \in C_{q}^{\varpi}(\Gamma)$, $B_{2 N}$ of system (11)-(15) and solutions $\rho_{1}(s), \rho_{2}(s) \in C_{q}^{\varpi}(\Gamma), B_{2 N}$ of system (18)-(22). This correspondence is given by (16) and (17).

3. Regularization. Investigation of the regularized system. We solve Eq. (18) for $\rho_{1}(s)$ and Eq. (19) for $\rho_{2}(s)$ assuming that $F_{1}(s), F_{2}(s)$ are given functions. Using the results of [2, 6], one can prove the following assertion (see Section 5).

Lemma 2. Let $F_{1}(s)$ and $F_{2}(s)$ be given Hölder functions on $\Gamma$. Then Eqs. (18) and (19) have the solutions in space $C_{q}^{\varpi}(\Gamma), \varpi \in(0,1], q \in[0,1)$. These solutions are given 
by the following expressions: for Eq. (18)

$$
\begin{gathered}
\rho_{1}(s)=\frac{\left(\sqrt{1+\beta^{2}}-\beta\right)}{2 \sqrt{1+\beta^{2}}} F_{1}(s) \\
+\frac{1}{2 \pi \sqrt{1+\beta^{2}} Q_{1}(s)} \int_{\Gamma} \frac{F_{1}(\sigma) Q_{1}(\sigma)}{\sigma-s} d \sigma-\frac{\cos \pi \eta}{Q_{1}(s)} \sum_{m=0}^{N-1} B_{m} s^{m}, \quad s \in \Gamma,
\end{gathered}
$$

for Eq. (19)

$$
\begin{gathered}
\rho_{2}(s)=\frac{\left(\sqrt{1+\beta^{2}}+\beta\right)}{2 \sqrt{1+\beta^{2}}} F_{2}(s) \\
-\frac{1}{2 \pi \sqrt{1+\beta^{2}} Q_{2}(s)} \int_{\Gamma} \frac{F_{2}(\sigma) Q_{2}(\sigma)}{\sigma-s} d \sigma-\frac{\sin \pi \eta}{Q_{2}(s)} \sum_{m=0}^{N-1} B_{m+N} s^{m}, \quad s \in \Gamma,
\end{gathered}
$$

where $B_{0}, \ldots, B_{2 N-1}$ are arbitrary real constants,

$$
\begin{gathered}
Q_{1}(s)=\prod_{n=1}^{N}\left|s-a_{n}\right|^{1 / 2-\eta}\left|s-b_{n}\right|^{1 / 2+\eta} \operatorname{sign}\left(s-a_{n}\right), \\
Q_{2}(s)=\prod_{n=1}^{N}\left|s-a_{n}\right|^{1-\eta}\left|s-b_{n}\right|^{\eta} \operatorname{sign}\left(s-a_{n}\right) .
\end{gathered}
$$

The number $\eta$ is defined by

$$
\eta=\frac{1}{2 \pi} \operatorname{arcctg} \beta \in(0,1 / 2)
$$

and satisfies the following relations:

$$
\begin{gathered}
\cos \pi \eta=\left(\frac{\sqrt{1+\beta^{2}}+\beta}{2 \sqrt{1+\beta^{2}}}\right)^{1 / 2}, \quad \sin \pi \eta=\left(\frac{\sqrt{1+\beta^{2}}-\beta}{2 \sqrt{1+\beta^{2}}}\right)^{1 / 2}, \\
\operatorname{tg} \pi \eta=\left(\sqrt{1+\beta^{2}}-\beta\right), \quad \operatorname{ctg} \pi \eta=\left(\sqrt{1+\beta^{2}}+\beta\right) .
\end{gathered}
$$

Substituting the expressions for $F_{1}(s)$ and $F_{2}(s)$ into formulas for $\rho_{1}(s)$ and $\rho_{2}(s)$, we obtain the system of regularized equations

$$
\begin{gathered}
\rho_{1}(s)+\frac{1}{Q_{1}(s)} \int_{\Gamma} \rho_{1}(\sigma) K_{11}(s, \sigma) d \sigma+\frac{1}{Q_{1}(s)} \int_{\Gamma} \rho_{2}(\sigma) K_{12}(s, \sigma) d \sigma \\
+\cos \pi \eta \frac{\sum_{m=0}^{N-1} B_{m} s^{m}}{Q_{1}(s)}=\frac{\Phi_{1}(s)}{Q_{1}(s)}, \quad s \in \Gamma, \\
\rho_{2}(s)+\frac{1}{Q_{2}(s)} \int_{\Gamma} \rho_{1}(\sigma) K_{21}(s, \sigma) d \sigma+\frac{1}{Q_{2}(s)} \int_{\Gamma} \rho_{2}(\sigma) K_{22}(s, \sigma) d \sigma \\
+\sin \pi \eta \frac{\sum_{m=0}^{N-1} B_{m+N} s^{m}}{Q_{2}(s)}=\frac{\Phi_{2}(s)}{Q_{2}(s)}, \quad s \in \Gamma,
\end{gathered}
$$


where we have used the notations

$$
\begin{gathered}
K_{11}(s, \sigma)=-\frac{Y_{2}(s, \sigma) Q_{1}(s)}{2 \sqrt{1+\beta^{2}}}-\frac{\left(\sqrt{1+\beta^{2}}+\beta\right)}{2 \pi \sqrt{1+\beta^{2}}} \int_{\Gamma} \frac{Y_{2}(\xi, \sigma)}{\xi-s} Q_{1}(\xi) d \xi, \\
K_{12}(s, \sigma)=\frac{Y_{1}(s, \sigma) Q_{1}(s)}{2 \sqrt{1+\beta^{2}}}+\frac{\left(\sqrt{1+\beta^{2}}+\beta\right)}{2 \pi \sqrt{1+\beta^{2}}} \int_{\Gamma} \frac{Y_{1}(\xi, \sigma)}{\xi-s} Q_{1}(\xi) d \xi, \\
K_{21}(s, \sigma)=\frac{Y_{1}(s, \sigma) Q_{2}(s)}{2 \sqrt{1+\beta^{2}}}-\frac{\left(\sqrt{1+\beta^{2}}-\beta\right)}{2 \pi \sqrt{1+\beta^{2}}} \int_{\Gamma} \frac{Y_{1}(\xi, \sigma)}{\xi-s} Q_{2}(\xi) d \xi, \\
K_{22}(s, \sigma)=\frac{Y_{2}(s, \sigma) Q_{2}(s)}{2 \sqrt{1+\beta^{2}}}-\frac{\left(\sqrt{1+\beta^{2}}-\beta\right)}{2 \pi \sqrt{1+\beta^{2}}} \int_{\Gamma} \frac{Y_{2}(\xi, \sigma)}{\xi-s} Q_{2}(\xi) d \xi, \\
\Phi_{1}(s)=\left(\sqrt{1+\beta^{2}}-\beta\right)\left(f^{\prime}+(s)+\frac{f^{-}}{\sqrt{1+\beta^{2}}}\right) Q_{1}(s) \\
+\frac{1}{\pi} \int_{\Gamma} \frac{Q_{1}(\sigma)}{\sigma-s}\left(f^{\prime}+(\sigma)+\frac{f^{-}(\sigma)}{\sqrt{1+\beta^{2}}}\right) d \sigma, \\
\Phi_{2}(s)=\left(f^{\prime}+(s)-\frac{f^{-}(s)}{\sqrt{1+\beta^{2}}}\right) Q_{2}(s) \\
-\frac{\left(\sqrt{1+\beta^{2}}-\beta\right)}{\pi} \int_{\Gamma} \frac{Q_{2}(\sigma)}{\sigma-s}\left(f^{\prime}+(\sigma)-\frac{f^{-}(\sigma)}{\sqrt{1+\beta^{2}}}\right) d \sigma .
\end{gathered}
$$

We put $\eta_{0}=\min \{\eta, 1 / 2-\eta\}$. Note that the densities of singular integrals in the expressions for $K_{p j}(s, \sigma)$ and $\Phi_{p}(s) \quad(p, j=1,2)$ satisfy the Hölder condition on $\Gamma$. (The densities in $K_{p j}(s, \sigma)$ satisfy the Hölder condition with respect to both variables.) In particular, these densities satisfy the Hölder condition with respect to the variable $\xi$ on $\Gamma$ with the exponent $\omega=\min \{\lambda, \eta, 1 / 2-\eta\}$ (uniformly with respect to $\sigma$ in the case of $K_{p j}(s, \sigma)$ ), because $Q_{1}(\xi), Q_{2}(\xi)$ belong to $C^{0, \eta_{0}}(\Gamma)$. These densities vanish if $\xi$ is the endpoint of $\Gamma$ because $Q_{1}(\xi), Q_{2}(\xi)$ vanish at the endpoints of $\Gamma$.

This, together with properties of singular integrals [6, Sec. 18] imply the following assertion.

Lemma 3. The functions $K_{p j}(s, \sigma)(p=1,2, j=1,2)$ satisfy the Hölder condition on $\Gamma$ with respect to both variables. In particular, these functions satisfy the Hölder condition on $\Gamma$ with respect to $s$ with the exponent $\omega=\min \{\lambda, \eta, 1 / 2-\eta\}$ uniformly with respect to $\sigma \in \Gamma$. If conditions (7) are satisfied, then $\Phi_{1}(s), \Phi_{2}(s) \in C^{0, \omega}(\Gamma)$.

Obviously, if functions $\rho_{1}(s)$ and $\rho_{2}(s)$ from the space $C_{q}^{\varpi}(\Gamma)$ with $\varpi \in(0,1]$ and $q \in[0,1)$ satisfy integral equations (23) and (24), then these functions can be represented in the form $\rho_{j}(s)=\rho_{j *}(s) / Q_{j}(s), j=1,2$ where $\rho_{1 *}(s), \rho_{2 *}(s) \in C^{0, \omega}(\Gamma), \omega$ is given by Lemma 3. So, from here on, the functions $\rho_{1}(s)$ and $\rho_{2}(s)$ are looked for in this form. It 
follows from this representation that $\rho_{1}(s), \rho_{2}(s) \in C_{q}^{\varpi}(\Gamma)$, where

$$
q=\max \{1 / 2+\eta, 1-\eta\}, \quad \varpi=\left\{\begin{array}{cc}
\min \{\omega,|2 \eta-1 / 2|\}, & \eta \neq 1 / 4, \\
\omega, & \eta=1 / 4 .
\end{array}\right.
$$

Below we assume that $\omega=\min \{\lambda, \eta, 1 / 2-\eta\} ; \varpi$ and $q$ are defined in (27). Note that $0<\omega \leq 1 / 4,0<\varpi \leq 1 / 4,1 / 2<q<1$. If $\beta>0$, then $0<\eta<1 / 4$ and $q=1-\eta$; if $\beta<0$, then $1 / 4<\eta<1 / 2$ and $q=1 / 2+\eta$; and if $\beta=0$, then $\eta=1 / 4, q=3 / 4$.

We multiply Eq. (23) by $Q_{1}(s)$, and Eq. (24) by $Q_{2}(s)$. As new unknown functions, we choose $\rho_{j *}(s)=\rho_{j}(s) Q_{j}(s) \in C^{0, \omega}(\Gamma), \quad j=1,2$. Note that there is a one-to-one correspondence between $\rho_{j}(s)$ and $\rho_{j *}(s) \quad(j=1,2)$. Equations (23) and (24) acquire the form

$$
\begin{gathered}
\rho_{1 *}(s)+\int_{\Gamma} \rho_{1 *}(\sigma) Q_{1}^{-1}(\sigma) K_{11}(s, \sigma) d \sigma+\int_{\Gamma} \rho_{2 *}(\sigma) Q_{2}^{-1}(\sigma) K_{12}(s, \sigma) d \sigma \\
\quad+\cos \pi \eta \sum_{m=0}^{N-1} B_{m} s^{m}=\Phi_{1}(s), \quad s \in \Gamma, \\
\rho_{2 *}(s)+\int_{\Gamma} \rho_{1 *}(\sigma) Q_{1}^{-1}(\sigma) K_{21}(s, \sigma) d \sigma+\int_{\Gamma} \rho_{2 *}(\sigma) Q_{2}^{-1}(\sigma) K_{22}(s, \sigma) d \sigma \\
+\sin \pi \eta \sum_{m=0}^{N-1} B_{m+N} s^{m}=\Phi_{2}(s), \quad s \in \Gamma .
\end{gathered}
$$

Using Lemma 3, one can easily prove the following assertion.

Lemma 4. Let the functions $\rho_{1 *}(s), \rho_{2 *}(s) \in C^{0}(\Gamma)$ satisfy Eqs. (28) and (29) for $\Phi_{1}(s), \Phi_{2}(s) \in C^{0, \omega}(\Gamma)$ with $\omega=\min \{\lambda, \eta, 1 / 2-\eta\}$. Then $\rho_{1 *}(s), \rho_{2 *}(s) \in C^{0, \omega}(\Gamma)$.

Remark. Obviously, if $\Phi_{1}(s), \Phi_{2}(s) \in C^{0, \omega_{1}}(\Gamma), \omega_{1} \in(0,1]$, then the functions $\rho_{1 *}(s), \rho_{2 *}(s)$ from $C^{0}(\Gamma)$, satisfying Eqs. (28) and (29), belong to the space $C^{0, \omega_{2}}(\Gamma)$ with $\omega_{2}=\min \left\{\lambda, \omega_{1}, \eta, 1 / 2-\eta\right\}$.

It follows from Lemma 3 that the condition $\Phi_{1}(s), \Phi_{2}(s) \in C^{0, \omega}(\Gamma)$ is satisfied if condition (7) is valid. Thus, further on we construct solutions $\rho_{1 *}(s)$ and $\rho_{2 *}(s)$ of Eqs. (28), (29) in $C^{0}(\Gamma)$. By Lemma 4, these solutions necessarily belong to $C^{0, \omega}(\Gamma)$. The solvability of Eqs. (28) and (29) in $C^{0}(\Gamma)$ can be analyzed under weaker conditions imposed on $\Phi_{1}(s), \Phi_{2}(s)$. More precisely, we assume that $\Phi_{1}(s), \Phi_{2}(s) \in C^{0}(\Gamma)$. If Eqs. (28) and (29) have solutions $\rho_{1 *}(s), \rho_{2 *}(s) \in C^{0}(\Gamma)$ for $\Phi_{1}(s), \Phi_{2}(s) \in C^{0, \omega}(\Gamma) \subset C^{0}(\Gamma)$, then, by Lemma $4, \rho_{1 *}(s), \rho_{2 *}(s) \in C^{0, \omega}(\Gamma)$.

We introduce operators $\mathbf{K}_{p j}$ by the formula

$$
\mathbf{K}_{p j}[v](s)=\int_{\Gamma} K_{p j}(s, \sigma) Q_{j}^{-1}(\sigma) v(\sigma) d \sigma, \quad p=1,2, j=1,2 .
$$

Lemma 5. The operators $\mathbf{K}_{p j}(p=1,2, j=1,2)$ are compact operators from $C^{0}(\Gamma)$ to $C^{0}(\Gamma)$.

The proof is performed by straightforward verification on the basis of the Arzela-Ascoli theorem and Lemma 3. 
Substituting the functions $\rho_{1}(s)=\rho_{1 *}(s) / Q_{1}(s)$ and $\rho_{2}(s)=\rho_{2 *}(s) / Q_{2}(s)$ into conditions (20)-(22), where $\rho_{1 *}(s)$ and $\rho_{2 *}(s)$ are given by (28), (29), we rewrite these conditions as follows:

$$
\sum_{j=1}^{2} \int_{\Gamma} Q_{j}^{-1}(\sigma) L_{n, j}(\sigma) \rho_{j *}(\sigma) d \sigma+\sum_{m=0}^{2 N} w_{n, m} B_{m}=\chi_{n}, \quad n=0, \ldots, 2 N
$$

here we have used the notation

$$
\begin{aligned}
& \chi_{0}=\int_{\Gamma}\left(\frac{\Phi_{2}(s)}{Q_{2}(s)}-\left(\sqrt{1+\beta^{2}}+\beta\right) \frac{\Phi_{1}(s)}{Q_{1}(s)}\right) d s+4 \pi A \sqrt{1+\beta^{2}}, \\
& \chi_{n}=\int_{\Gamma_{n}}\left(\left(\sqrt{1+\beta^{2}}+\beta\right) \frac{\Phi_{2}(s)}{Q_{2}(s)}+\frac{\Phi_{1}(s)}{Q_{1}(s)}\right) d s, \quad n=1, \ldots, N, \\
& \chi_{n}=-2 \sqrt{1+\beta^{2}} f^{+}\left(a_{n-N}\right)+V\left[\frac{\Phi_{2}(\cdot)}{Q_{2}(\cdot)}-\left(\sqrt{1+\beta^{2}}+\beta\right) \frac{\Phi_{1}(\cdot)}{Q_{1}(\cdot)}\right]\left(x\left(a_{n-N}\right)\right) \\
& +T\left[\left(\sqrt{1+\beta^{2}}+\beta\right) \frac{\Phi_{2}(\cdot)}{Q_{2}(\cdot)}+\frac{\Phi_{1}(\cdot)}{Q_{1}(\cdot)}\right]\left(x\left(a_{n-N}\right)\right), \quad n=N+1, \ldots, 2 N, \\
& w_{0, m}=-\cos \pi \eta\left(\sqrt{1+\beta^{2}}+\beta\right) \int_{\Gamma} \frac{s^{m}}{Q_{1}(s)} d s, \quad m=0, \ldots, N-1, \\
& w_{0, m}=\sin \pi \eta \int_{\Gamma} \frac{s^{m-N}}{Q_{2}(s)} d s, \quad m=N, \ldots, 2 N-1, \\
& w_{n, m}=\cos \pi \eta \int_{\Gamma_{n}} \frac{s^{m}}{Q_{1}(s)} d s, \quad m=0, \ldots, N-1, \quad n=1, \ldots, N, \\
& w_{n, m}=\cos \pi \eta \int_{\Gamma_{n}} \frac{s^{m-N}}{Q_{2}(s)} d s, \quad m=N, \ldots, 2 N-1, \quad n=1, \ldots, N, \\
& w_{n, m}=\cos \pi \eta\left(-\left(\sqrt{1+\beta^{2}}+\beta\right) V\left[\frac{(\cdot)^{m}}{Q_{1}(\cdot)}\right]\left(x\left(a_{n-N}\right)\right)+T\left[\frac{(\cdot)^{m}}{Q_{1}(\cdot)}\right]\left(x\left(a_{n-N}\right)\right)\right) \text {, } \\
& m=0, \ldots, N-1, \quad n=N+1, \ldots, 2 N, \\
& w_{n, m}=\sin \pi \eta\left(V\left[\frac{(\cdot)^{m-N}}{Q_{2}(\cdot)}\right]\left(x\left(a_{n-N}\right)\right)+\left(\sqrt{1+\beta^{2}}+\beta\right) T\left[\frac{(\cdot)^{m-N}}{Q_{2}(\cdot)}\right]\left(x\left(a_{n-N}\right)\right)\right), \\
& m=N, \ldots, 2 N-1, \quad n=N+1, \ldots, 2 N \text {, } \\
& w_{0,2 N}=0, \quad w_{n, 2 N}=0, \quad n=1, \ldots, N, \\
& w_{n, 2 N}=-2 \sqrt{1+\beta^{2}}, \quad n=N+1, \ldots, 2 N, \\
& L_{0, j}(\sigma)=\int_{\Gamma}\left(\frac{K_{2 j}(s, \sigma)}{Q_{2}(s)}-\left(\sqrt{1+\beta^{2}}+\beta\right) \frac{K_{1 j}(s, \sigma)}{Q_{1}(s)}\right) d s, \quad j=1,2,
\end{aligned}
$$




$$
\begin{aligned}
& L_{n, j}(\sigma)=\int_{\Gamma_{n}}\left(\left(\sqrt{1+\beta^{2}}+\beta\right) \frac{K_{2 j}(s, \sigma)}{Q_{2}(s)}+\frac{K_{1 j}(s, \sigma)}{Q_{1}(s)}\right) d s, \quad j=1,2, \quad n=1, \ldots, N, \\
& L_{n, j}(\sigma)=V\left[\frac{K_{2 j}(\cdot, \sigma)}{Q_{2}(\cdot)}-\left(\sqrt{1+\beta^{2}}+\beta\right) \frac{K_{1 j}(\cdot, \sigma)}{Q_{1}(\cdot)}\right]\left(x\left(a_{n-N}\right)\right) \\
& +T\left[\left(\sqrt{1+\beta^{2}}+\beta\right) \frac{K_{2 j}(\cdot, \sigma)}{Q_{2}(\cdot)}+\frac{K_{1 j}(\cdot, \sigma)}{Q_{1}(\cdot)}\right]\left(x\left(a_{n-N}\right)\right), \quad j=1,2, \quad n=N+1, \ldots, 2 N .
\end{aligned}
$$

The dot $(\cdot)$ stands for the integration variable in the potentials. In system (31), the index $n=0$ corresponds to Eq. (21), the indices $n=1, \ldots, N$ correspond to Eq. (20), and $n=N+1, \ldots, 2 N$ correspond to (22). For each $n$, in all formulas containing the angular potential, we choose the same branch of the function $\psi\left(x\left(a_{n-N}\right), y(s)\right)$, where $s \in \Gamma$. Alternatively, one can use the representation of the angular potential in terms of the double-layer potential.

Note that the functions $Q_{1}(s)$ and $Q_{2}(s)$ can readily be reduced to a form admitting an analytic extension to the entire complex plane (see Sections 5 and 6 below). By applying residue theory (see Section 6) to these functions, one can readily obtain the relations

$$
\cos \pi \eta \int_{\Gamma} \frac{s^{m}}{Q_{1}(s)} d s=\sin \pi \eta \int_{\Gamma} \frac{s^{m}}{Q_{2}(s)} d s= \begin{cases}0, & m=0, \ldots, N-2, \\ \pi, & m=N-1 .\end{cases}
$$

Since the function $s^{m}(\cos \pi \eta) / Q_{1}(s)$ satisfies the homogeneous equation (18) and the function $s^{m}(\sin \pi \eta) / Q_{2}(s)$ satisfies the homogeneous equation (19) with $m=0,1, \ldots, N-1$, it follows (see Section 6) that

$$
\begin{gathered}
\int_{\Gamma} \frac{\Phi_{p}(s)}{Q_{p}(s)} d s=0, \quad p=1,2, \\
\int_{\Gamma} \frac{K_{p j}(s, \sigma)}{Q_{p}(s)} d s \equiv 0, \quad \sigma \in \Gamma, \quad p=1,2, \quad j=1,2 .
\end{gathered}
$$

Hence we have the following simplifications of the expressions for the coefficients of system (31):

$$
\begin{gathered}
w_{0, m}=0, \quad m=0,1, \ldots, N-2, N, N+1, \ldots, 2 N-2, \\
w_{0, N-1}=-\pi\left(\sqrt{1+\beta^{2}}+\beta\right), \quad w_{0,2 N-1}=\pi, \\
L_{0, j}(\sigma) \equiv 0, \quad \sigma \in \Gamma, \quad j=1,2, \quad \chi_{0}=4 \pi A \sqrt{1+\beta^{2}} .
\end{gathered}
$$

We introduce the column vector of coefficients: $\bar{B}=\left(B_{0}, \ldots, B_{2 N}\right)^{T} \in E_{2 N+1}$. System (28), (29), (31) can be rewritten in the form of a single equation for the unknown column vector $\bar{\rho}=\left(\rho_{1 *}(s), \rho_{2 *}(s), \bar{B}\right)^{T}$ from the Banach space $C^{0}(\Gamma) \times C^{0}(\Gamma) \times E_{2 N+1}$ with the norm $\|\bar{\rho}\|_{C^{0}(\Gamma) \times C^{0}(\Gamma) \times E_{2 N+1}}=\left\|\rho_{1 *}\right\|_{C^{0}(\Gamma)}+\left\|\rho_{2 *}\right\|_{C^{0}(\Gamma)}+\|\bar{B}\|_{E_{2 N+1}}$; that is,

$$
(\mathbf{I}+\mathbf{R}) \bar{\rho}=\bar{\Phi},
$$

where we have introduced the following notation:

$$
\bar{\Phi}=\left(\Phi_{1}(s), \Phi_{2}(s), \chi_{0}, \chi_{1}, \ldots, \chi_{2 N}\right)^{T} \in C^{0}(\Gamma) \times C^{0}(\Gamma) \times E_{2 N+1},
$$


where $\Phi_{1}(s)$ and $\Phi_{2}(s)$ are the functions given in (25), (26); $\mathbf{I}$ is the identity operator mapping the space $C^{0}(\Gamma) \times C^{0}(\Gamma) \times E_{2 N+1}$ into itself,

$$
\mathbf{R}=\left(\begin{array}{ccc}
\mathbf{K}_{11} & \mathbf{K}_{12} & P_{1} \\
\mathbf{K}_{21} & \mathbf{K}_{22} & P_{2} \\
\mathbf{L}_{1} & \mathbf{L}_{2} & W-I_{2 N+1}
\end{array}\right)
$$

$I_{2 N+1}$ is the identity matrix of order $2 N+1, W=\left\{w_{n, m}\right\}_{m=0, \ldots, 2 N}^{n=0, \ldots, 2 N}$ is a square matrix of order $(2 N+1) \times(2 N+1)$;

$$
\mathbf{L}_{j}=\left(\mathcal{L}_{0, j}, \mathcal{L}_{1, j}, \ldots, \mathcal{L}_{2 N, j}\right)^{T}, \quad j=1,2 ;
$$

$\mathcal{L}_{n, j}$ are the functionals expressed in terms of the functions $L_{n, j}(\sigma)$ by the formulas

$$
\mathcal{L}_{n, j} \rho_{j *}=\int_{\Gamma} Q_{j}^{-1}(\sigma) L_{n, j}(\sigma) \rho_{j *}(\sigma) d \sigma, \quad j=1,2, \quad n=0, \ldots, 2 N ;
$$

$\mathbf{K}_{p j}(p=1,2, j=1,2)$ are the operators given by (30); $P_{1}$ and $P_{2}$ are the multiplication operators defined by the relations

$$
P_{1} \bar{B}=\cos \pi \eta \sum_{m=0}^{N-1} B_{m} s^{m}, \quad P_{2} \bar{B}=\sin \pi \eta \sum_{m=0}^{N-1} B_{m+N} s^{m} .
$$

Lemma 6. Eq. (32) is a Fredholm equation of the second kind and index zero in the space $C^{0}(\Gamma) \times C^{0}(\Gamma) \times E_{2 N+1}$.

Proof. Consider the operators comprising the operator R. By Lemma $5, \mathbf{K}_{p j} \quad(p=$ $1,2, j=1,2)$ are compact operators from $C^{0}(\Gamma)$ into $C^{0}(\Gamma)$. The operator $\left(W-I_{2 N+1}\right)$ maps $E_{2 N+1}$ into $E_{2 N+1}$. Since any linear operator acting in a finite-dimensional space is a compact operator [7, it follows that $\left(W-I_{2 N+1}\right)$ is a compact operator. The operators $P_{1}$ and $P_{2}$ mapping $E_{2 N+1}$ into $C^{0}(\Gamma)$ and the operators $\mathbf{L}_{1}$ and $\mathbf{L}_{2}$ mapping $C^{0}(\Gamma)$ into $E_{2 N+1}$ are finite-dimensional and therefore compact [7, 8, p. 64]. Thus, all operators comprising $\mathbf{R}$ are compact operators. Therefore, $\mathbf{R}$ is a compact operator from $C^{0}(\Gamma) \times C^{0}(\Gamma) \times E_{2 N+1}$ into itself. By [8, p. 67], an equation of the form (32) with compact operator $\mathbf{R}$ satisfies the Fredholm alternative. The proof of the lemma is complete.

Lemma 7. Let condition (7) be satisfied, and let Eq. (32) have a solution

$$
\bar{\rho}=\left(\rho_{1 *}(s), \rho_{2 *}(s), \bar{B}\right)^{T} \in C^{0, \omega}(\Gamma) \times C^{0, \omega}(\Gamma) \times E_{2 N+1},
$$

where $\omega=\min \{\lambda, \eta, 1 / 2-\eta\}$. Then

(1) the functions

$$
\rho_{1}(s)=\frac{\rho_{1 *}(s)}{Q_{1}(s)} \in C_{q}^{\varpi}(\Gamma), \quad \rho_{2}(s)=\frac{\rho_{2 *}(s)}{Q_{2}(s)} \in C_{q}^{\varpi}(\Gamma)
$$

and the constant $B_{2 N}$ (that is, the last component of the vector $\bar{B}$ ) solve the system (18)-(22), where the indices $\varpi$ and $q$ are given by (27); 
(2) the solution of Problem $\mathcal{S}$ exists and is given by (10), where

$$
\begin{gathered}
\nu(s)=\frac{\rho_{1}(s)+\left(\sqrt{1+\beta^{2}}+\beta\right) \rho_{2}(s)}{2 \sqrt{1+\beta^{2}}} \\
=\frac{1}{2 \sqrt{1+\beta^{2}}}\left[\frac{\rho_{1 *}(s)}{Q_{1}(s)}+\left(\sqrt{1+\beta^{2}}+\beta\right) \frac{\rho_{2 *}(s)}{Q_{2}(s)}\right] \in C_{q}^{\varpi}(\Gamma), \\
\mu(s)=\frac{\rho_{2}(s)-\left(\sqrt{1+\beta^{2}}+\beta\right) \rho_{1}(s)}{2 \sqrt{1+\beta^{2}}} \\
=\frac{1}{2 \sqrt{1+\beta^{2}}}\left[\frac{\rho_{2 *}(s)}{Q_{2}(s)}-\left(\sqrt{1+\beta^{2}}+\beta\right) \frac{\rho_{1 *}(s)}{Q_{1}(s)}\right] \in C_{q}^{\varpi}(\Gamma),
\end{gathered}
$$

and $B_{2 N}$ is the last component of the vector $\bar{B}$.

Proof. Let Eq. (32) have a solution

$$
\bar{\rho}=\left(\rho_{1 *}(s), \rho_{2 *}(s), \bar{B}\right)^{T} \in C^{0, \omega}(\Gamma) \times C^{0, \omega}(\Gamma) \times E_{2 N+1},
$$

transforming Eqs. (28), (29) and (31) into identities. Let us prove assertion (1). Obviously, the functions $\rho_{1}(s), \rho_{2}(s)$ constructed by formulas (33) and the constants $B_{0}, \ldots, B_{2 N-1}$ comprising the vector $\bar{B}$ convert Eqs. (23) and (24) into identities. We introduce singular operators $\mathbf{S}_{-}$and $\mathbf{S}_{+}$, acting on functions $w(s) \in C_{q}^{\varpi}(\Gamma)$ as follows:

$$
\begin{aligned}
& \mathbf{S}_{-} w=w(s)-\left(\sqrt{1+\beta^{2}}+\beta\right) \frac{1}{\pi} \int_{\Gamma} \frac{w(\sigma)}{\sigma-s} d \sigma, \\
& \mathbf{S}_{+} w=w(s)+\left(\sqrt{1+\beta^{2}}-\beta\right) \frac{1}{\pi} \int_{\Gamma} \frac{w(\sigma)}{\sigma-s} d \sigma .
\end{aligned}
$$

We apply the operator $\mathbf{S}_{-}$to identity (23) and the operator $\mathbf{S}_{+}$to identity (24). As a result, we find that $\rho_{1}(s)$ and $\rho_{2}(s)$ satisfy singular integral equations (18) and (19). Using identities (31), which are components of identity (32), one can readily see that the functions $\rho_{1}(s)$ and $\rho_{2}(s)$ and the constant $B_{2 N}$ satisfy conditions (20)-(22). Therefore, the functions (33) and the constant $B_{2 N}$ solve system (18)-(22), and the proof of the first assertion is complete.

(2) As shown in item (1), the functions given by (33) and the constant $B_{2 N}$ solve system (18)-(22). By Lemma 1, the functions $\mu(s)$ and $\nu(s)$ given by (17) (or, which is the same, by (34)) and the constant $B_{2 N}$ solve system (11)-(15). We substitute the functions $\mu(s)$ and $\nu(s)$ and the constant $B_{2 N}$ into the function (10). By Theorem 2, the function (10) is a solution of Problem $\mathcal{S}$. The proof of the lemma is complete.

In the following section, we prove the solvability of Eq. (32) and Problem $\mathcal{S}$.

4. Existence of a solution. Let us now prove the solvability of Eq. (32).

Theorem 3. (1) Eq. (32) has a unique solution $\bar{\rho}$ in the space $C^{0}(\Gamma) \times C^{0}(\Gamma) \times E_{2 N+1}$ for an arbitrary right-hand side

$$
\bar{\Phi} \in C^{0}(\Gamma) \times C^{0}(\Gamma) \times E_{2 N+1} .
$$

(2) If the right-hand side $\bar{\Phi}$ belongs to the space $C^{0, \omega}(\Gamma) \times C^{0, \omega}(\Gamma) \times E_{2 N+1}, \omega=$ $\min \{\lambda, \eta, 1 / 2-\eta\}$, then the solution $\bar{\rho}$ belongs to the same space. 
Proof. (1) By Lemma 6, Eq. (32) is a Fredholm equation in the space $C^{0}(\Gamma) \times C^{0}(\Gamma) \times$ $E_{2 N+1}$. By the Fredholm alternative, to prove assertion (1), it suffices to show that the homogeneous equation (32) has only the trivial solution in this space. We prove the latter assertion by a contradiction. Suppose that the homogeneous equation (32) has a nontrivial solution $\bar{\rho}^{0}=\left(\rho_{1 *}^{0}(s), \rho_{2 *}^{0}(s), B_{0}^{0}, \ldots, B_{2 N}^{0}\right)^{T} \in C^{0}(\Gamma) \times C^{0}(\Gamma) \times E_{2 N+1}$. This implies that the functions $\rho_{1 *}^{0}(s), \rho_{2 *}^{0}(s) \in C^{0}(\Gamma)$ and the constants $B_{0}^{0}, \ldots, B_{2 N-1}^{0}$ satisfy the homogeneous equations (28), (29), which are part of the vector equation (32). By Lemma 4, one can claim that $\bar{\rho}^{0} \in C^{0, \omega}(\Gamma) \times C^{0, \omega}(\Gamma) \times E_{2 N+1}$. The homogeneous equation (32) occurs if $f^{+}(s) \equiv 0, f^{-}(s) \equiv 0$ and $A=0$, i.e., the homogeneous problem $\mathcal{S}$ leads to the homogeneous equation (32). By assertion 2 of Lemma 7, the function

$$
u^{0}(x)=V\left[\mu^{0}\right](x)+T\left[\nu^{0}\right](x)+B_{2 N}^{0}
$$

is a solution of the homogeneous problem $\mathcal{S}$; here $\mu^{0}$ and $\nu^{0}$ are defined via $\rho_{1 *}^{0}$ and $\rho_{2 *}^{0}$ by formulas (34). On the other hand, it follows from Theorem 1 that the homogeneous problem $\mathcal{S}$ has only the trivial solution

$$
u^{0}(x) \equiv 0 .
$$

Taking into account the limit formulas for potentials [3, 4,

$$
\begin{aligned}
& {\left.\left[\left(\frac{\partial V[\mu]}{\partial \mathbf{n}_{x}}\right)^{+}-\left(\frac{\partial V[\mu]}{\partial \mathbf{n}_{x}}\right)^{-}\right]\right|_{\Gamma}=\mu(s),\left.\quad\left[\left(\frac{\partial T[\nu]}{\partial \mathbf{n}_{x}}\right)^{+}-\left(\frac{\partial T[\nu]}{\partial \mathbf{n}_{x}}\right)^{-}\right]\right|_{\Gamma}=0, \quad s \in \Gamma,} \\
& {\left.\left[\left(\frac{\partial V[\mu]}{\partial \tau_{x}}\right)^{+}-\left(\frac{\partial V[\mu]}{\partial \tau_{x}}\right)^{-}\right]\right|_{\Gamma}=0,\left.\quad\left[\left(\frac{\partial T[\nu]}{\partial \tau_{x}}\right)^{+}-\left(\frac{\partial T[\nu]}{\partial \tau_{x}}\right)^{-}\right]\right|_{\Gamma}=\nu(s), \quad s \in \Gamma,}
\end{aligned}
$$

we obtain the relations

$$
\begin{aligned}
& {\left.\left[\left(\frac{\partial u^{0}}{\partial \tau_{x}}\right)^{+}-\left(\frac{\partial u^{0}}{\partial \tau_{x}}\right)^{-}\right]\right|_{x(s) \in \Gamma}=\nu^{0}(s) \equiv 0} \\
& {\left.\left[\left(\frac{\partial u^{0}}{\partial \mathbf{n}_{x}}\right)^{+}-\left(\frac{\partial u^{0}}{\partial \mathbf{n}_{x}}\right)^{-}\right]\right|_{x(s) \in \Gamma}=\mu^{0}(s) \equiv 0 .}
\end{aligned}
$$

By the formulas (34) (see also (16)), we have

$$
\begin{aligned}
& \frac{\rho_{1 *}^{0}(s)}{Q_{1}(s)}=\frac{\nu^{0}(s)-\left(\sqrt{1+\beta^{2}}+\beta\right) \mu^{0}(s)}{\left(\sqrt{1+\beta^{2}}+\beta\right)} \equiv 0, \quad s \in \Gamma, \\
& \frac{\rho_{2 *}^{0}(s)}{Q_{2}(s)}=\frac{\mu^{0}(s)+\left(\sqrt{1+\beta^{2}}+\beta\right) \nu^{0}(s)}{\left(\sqrt{1+\beta^{2}}+\beta\right)} \equiv 0, \quad s \in \Gamma .
\end{aligned}
$$

Thus, $\rho_{1 *}^{0}(s) \equiv 0, \rho_{2 *}^{0}(s) \equiv 0, s \in \Gamma$. Taking into account (36) and (35), we obtain $B_{2 N}^{0}=0$. From the homogeneous identities (28) and (29), which are part of Eq. (32), we obtain

$$
\sum_{m=0}^{N-1} B_{m}^{0} s^{m} \equiv 0, \quad \sum_{m=0}^{N-1} B_{m+N}^{0} s^{m} \equiv 0, \quad s \in \Gamma .
$$


By the main theorem of algebra on the number of roots of a polynomial, the identities are valid only if $B_{m}^{0}=0, m=0,1, \ldots, 2 N-1$. Thus, $\bar{\rho}^{0} \equiv 0$, which contradicts the above-stipulated assumption. The proof of assertion (1) is complete.

(2) Let $\bar{\rho}=\left(\rho_{1 *}(s), \rho_{2 *}(s), \bar{B}\right)^{T} \in C^{0}(\Gamma) \times C^{0}(\Gamma) \times E_{2 N+1}$ be a solution of the inhomogeneous equation (32) for $\bar{\Phi} \in C^{0, \omega}(\Gamma) \times C^{0, \omega}(\Gamma) \times E_{2 N+1}$. It follows from assertion (1) that this solution exists. The functions $\rho_{1 *}(s)$ and $\rho_{2 *}(s)$ satisfy Eqs. (28) and (29), which are part of Eq. (32). Consequently, by Lemma $4: \bar{\rho} \in C^{0, \omega}(\Gamma) \times C^{0, \omega}(\Gamma) \times E_{2 N+1}$.

The proof of the theorem is complete.

Therefore, Theorem 3 provides the unique solvability of Eq. (32) in the space $C^{0, \omega}(\Gamma) \times$ $C^{0, \omega}(\Gamma) \times E_{2 N+1}$ if the right-hand side of Eq. (32) belongs to the same space. By Lemma 3 , the vector $\bar{\Phi}$ does belong to this space if conditions (7) are satisfied. By assertion 2 of Lemma 7, Problem $\mathcal{S}$ has a solution given by (10), where $\mu(s), \nu(s), B_{2 N}$ can be expressed via the elements of a solution of (32). It turns out that $\mu(s), \nu(s) \in C_{q}^{\varpi}(\Gamma)$.

Using Theorem 1, Theorem 3 and assertion 2 of Lemma 7, we state the resulting assertion.

Theorem 4. Let $\Gamma \in C^{2, \lambda}$ and let conditions (7) be satisfied. Then the solution of Problem $\mathcal{S}$ exists, is unique and is given by (10), where $\mu(s)$ and $\nu(s)$ are given by (34); the functions $\rho_{1 *}(s), \rho_{2 *}(s) \in C^{0, \omega}(\Gamma) \quad(\omega=\min \{\lambda, \eta, 1 / 2-\eta\})$ and the constant $B_{2 N}$ can be found from the Fredholm equation of the second kind (32), which is uniquely solvable.

It follows from the properties of potentials [4 that, as a rule, the gradient of the solution of Problem $\mathcal{S}$ is unbounded in neighborhoods of the endpoints of the contour $\Gamma$. Moreover [4, Theorem 5], inequality (11) is valid with $\delta=-q$, where $q$ is the constant given by (27).

5. Solution of the singular equations. Let us prove Lemma 2. We consider the singular integral equation (19). Let the function $\rho_{2}(s)$ satisfy Eq. (19). We consider the Cauchy type integral

$$
R_{2}(z)=\frac{1}{2 \pi i} \int_{\Gamma} \frac{\rho_{2}(\sigma)}{\sigma-z} d \sigma, \quad z \notin \Gamma .
$$

By the Sokhotskii formulas, we have

$$
\begin{gathered}
R_{2}^{+}(s)-R_{2}^{-}(s)=\rho_{2}(s), \quad s \in \Gamma, \\
R_{2}^{+}(s)+R_{2}^{-}(s)=\frac{1}{\pi i} \int_{\Gamma} \frac{\rho_{2}(\sigma)}{\sigma-s} d \sigma, \quad s \in \Gamma .
\end{gathered}
$$

Therefore, if the function $\rho_{2}(s)$ satisfies Eq. (19), then the limit values of the function $R_{2}(z)$ on $\Gamma^{ \pm}$must satisfy the relation

$$
\left(R_{2}^{+}(s)-R_{2}^{-}(s)\right)+\left(\sqrt{1+\beta^{2}}-\beta\right) i\left(R_{2}^{+}(s)+R_{2}^{-}(s)\right)=F_{2}(s), \quad s \in \Gamma .
$$

By reducing the last equation to a standard form, we give a rigorous statement of the resulting conjunction problem [2]. 
Problem C. Find a function $R_{2}(z)$ that is piecewise holomorphic with jump line $\Gamma$ and satisfies the condition $R_{2}(\infty)=0$ as well as the boundary condition

$$
R_{2}^{+}(s)-\mathcal{G}(s) R_{2}^{-}(s)=\frac{F_{2}(s)}{1+i\left(\sqrt{1+\beta^{2}}-\beta\right)}, \quad s \in \Gamma,
$$

where

$$
\mathcal{G}(s)=\frac{\beta-i}{\sqrt{1+\beta^{2}}} .
$$

(The condition $R_{2}(\infty)=0$ follows from the form of the function (37).)

In the notion of the piecewise holomorphic function we include the following condition: a function may have integrable singularities at endpoints of the contour $\Gamma$.

Let us solve Problem $\mathrm{C}$ and then, using (38), find the function $\rho_{2}(s)$. This function will be a solution of the singular integral equation (19).

Let us define the number $\eta$ using the formula

$$
e^{i 2 \pi \eta}=\frac{\beta+i}{\sqrt{1+\beta^{2}}}, \quad \eta \in(0,1 / 2)
$$

(this definition is equivalent to that given in Lemma 2).

The canonical solution of the homogeneous conjunction Problem $\mathrm{C}$ has the form [2] $R_{2}^{0}(z)=1 / Q_{2}(z)$, where $Q_{2}(z)=\prod_{n=1}^{N}\left(z-a_{n}\right)^{1-\eta}\left(z-b_{n}\right)^{\eta}$.

Let us find the expressions for the limit values of $Q_{2}(z)$ on $\Gamma^{+}$and $\Gamma^{-}$:

$$
\lim _{z \rightarrow s \in \Gamma^{ \pm}} Q_{2}(z) \equiv Q_{2}^{ \pm}(s)=\prod_{n=1}^{N}\left[\left(s-a_{n}\right)^{1-\eta}\right]^{ \pm}\left[\left(s-b_{n}\right)^{\eta}\right]^{ \pm}
$$

If $s \notin \Gamma_{n}$, then $\left[\left(s-a_{n}\right)^{1-\eta}\left(s-b_{n}\right)^{\eta}\right]^{ \pm}=\left|s-a_{n}\right|^{1-\eta}\left|s-b_{n}\right|^{\eta} \operatorname{sign}\left(s-a_{n}\right)$. If $s \in \Gamma_{n}$, then $\left[\left(s-a_{n}\right)^{1-\eta}\right]^{ \pm}=\left|s-a_{n}\right|^{1-\eta},\left[\left(s-b_{n}\right)^{\eta}\right]^{+}=e^{i \pi \eta}\left|s-b_{n}\right|^{\eta}$, $\left[\left(s-b_{n}\right)^{\eta}\right]^{-}=e^{-i \pi \eta}\left|s-b_{n}\right|^{\eta}$. Here everywhere $n=1, \ldots, N$.

Therefore,

$$
Q_{2}^{+}(s)=e^{i \pi \eta} Q_{2}(s), \quad Q_{2}^{-}(s)=e^{-i \pi \eta} Q_{2}(s), \quad s \in \Gamma .
$$

Here $Q_{2}(s)=\prod_{n=1}^{N}\left|s-a_{n}\right|^{1-\eta}\left|s-b_{n}\right|^{\eta} \operatorname{sign}\left(s-a_{n}\right)$ is a real function called the direct value of the function $Q_{2}(z)$ on the axis $O s$, in particular, on $\Gamma$.

Let us return to the conjunction Problem $\mathrm{C}$ for the function $R_{2}(z)$. By [2], the solution of the inhomogeneous conjunction Problem $\mathrm{C}$ with the condition $R_{2}(\infty)=0$ has the form

$$
R_{2}(z)=\frac{1}{2 \pi i Q_{2}(z)} \int_{\Gamma} \frac{F_{2}(\sigma) Q_{2}^{+}(\sigma)}{(\sigma-z)\left(1+i\left(\sqrt{1+\beta^{2}}-\beta\right)\right)} d \sigma+\frac{\sum_{m=0}^{N-1} \widetilde{B}_{m+N} z^{m}}{Q_{2}(z)},
$$

where $\widetilde{B}_{N}, \ldots, \widetilde{B}_{2 N-1}$ are arbitrary complex constants. 
Now, by (38), we find a solution of Eq. (19):

$$
\begin{gathered}
\rho_{2}(s)=R_{2}^{+}(s)-R_{2}^{-}(s)=\frac{1}{Q_{2}^{+}(s)}\left(\frac{F_{2}(s) Q_{2}^{+}(s)}{2\left(1+i\left(\sqrt{1+\beta^{2}}-\beta\right)\right)}\right. \\
\left.+\frac{1}{2 \pi i} \int_{\Gamma} \frac{F_{2}(\sigma) Q_{2}^{+}(\sigma)}{1+i\left(\sqrt{1+\beta^{2}}-\beta\right)} \frac{d \sigma}{\sigma-s}+\sum_{m=0}^{N-1} \widetilde{B}_{m+N} s^{m}\right) \\
\quad-\frac{1}{Q_{2}^{-}(s)}\left(-\frac{F_{2}(s) Q_{2}^{+}(s)}{2\left(1+i\left(\sqrt{1+\beta^{2}}-\beta\right)\right)}\right. \\
\left.+\frac{1}{2 \pi i} \int_{\Gamma} \frac{F_{2}(\sigma) Q_{2}^{+}(\sigma)}{1+i\left(\sqrt{1+\beta^{2}}-\beta\right)} \frac{d \sigma}{\sigma-s}+\sum_{m=0}^{N-1} \widetilde{B}_{m+N} s^{m}\right) \\
=\frac{\left(\sqrt{1+\beta^{2}}+\beta\right)}{2 \sqrt{1+\beta^{2}}} F_{2}(s)-\frac{1}{2 \pi \sqrt{1+\beta^{2}} Q_{2}(s)} \int_{\Gamma} \frac{F_{2}(\sigma) Q_{2}(\sigma)}{\sigma-s} d \sigma-\sin \pi \eta \frac{\sum_{m=0}^{N-1} B_{m+N} s^{m}}{Q_{2}(s)}
\end{gathered}
$$

where $B_{N}, \ldots, B_{2 N-1}$ are arbitrary real constants. In the latter relation, we have used formulas (40) and the definition of the constant $\eta$. This completes the proof of Lemma 2 for (19).

The singular integral equation (18) for the function $\rho_{1}(s)$ can be treated in a similar way. For the function

$$
R_{1}(z)=\frac{1}{2 \pi i} \int_{\Gamma} \frac{\rho_{1}(\sigma)}{\sigma-z} d \sigma, \quad z \notin \Gamma,
$$

we obtain the conjunction problem [2] with the boundary condition

$$
R_{1}^{+}(s)+\mathcal{G}(s) R_{1}^{-}(s)=\frac{F_{1}(s)}{1-i\left(\sqrt{1+\beta^{2}}+\beta\right)}, \quad s \in \Gamma .
$$

The solution of the homogeneous conjunction problem has the form $R_{1}(z)=1 / Q_{1}(z)$, where $Q_{1}(z)=\prod_{n=1}^{N}\left(z-a_{n}\right)^{1 / 2-\eta}\left(z-b_{n}\right)^{1 / 2+\eta}$. The limit values of the function $Q_{1}(z)$ on $\Gamma^{ \pm}$are given by the relations

$$
Q_{1}^{+}(s)=i e^{i \pi \eta} Q_{1}(s), \quad Q_{1}^{-}(s)=-i e^{-i \pi \eta} Q_{1}(s), \quad s \in \Gamma,
$$

where $Q_{1}(s)=\prod_{n=1}^{N}\left|s-a_{n}\right|^{1 / 2-\eta}\left|s-b_{n}\right|^{1 / 2+\eta} \operatorname{sign}\left(s-a_{n}\right)$ is the direct value of $Q_{1}(z)$ on the axis $O s$, in particular, on $\Gamma$. We construct the solution of the inhomogeneous conjunction problem and obtain

$$
\begin{gathered}
\rho_{1}(s)=\frac{\left(\sqrt{1+\beta^{2}}-\beta\right)}{2 \sqrt{1+\beta^{2}}} F_{1}(s) \\
+\frac{1}{2 \pi \sqrt{1+\beta^{2}} Q_{1}(s)} \int_{\Gamma} \frac{F_{1}(\sigma) Q_{1}(\sigma)}{\sigma-s} d \sigma-\cos \pi \eta \frac{\sum_{m=0}^{N-1} B_{m} s^{m}}{Q_{1}(s)},
\end{gathered}
$$


where $B_{0}, \ldots, B_{N-1}$ are arbitrary real constants. The proof of Lemma 2 is complete.

6. Computation of integrals. Here we consider the integrals

$$
\begin{gathered}
I_{1, m}=\cos \pi \eta \int_{\Gamma} \frac{s^{m}}{Q_{1}(s)} d s, \quad I_{2, m}=\sin \pi \eta \int_{\Gamma} \frac{s^{m}}{Q_{2}(s)} d s, \quad m=0,1, \ldots, N-1, \\
J_{1, m}(s)=\cos \pi \eta \int_{\Gamma} \frac{\sigma^{m}}{Q_{1}(\sigma)} \frac{d \sigma}{\sigma-s}, \quad J_{2, m}(s)=\sin \pi \eta \int_{\Gamma} \frac{\sigma^{m}}{Q_{2}(\sigma)} \frac{d \sigma}{\sigma-s} .
\end{gathered}
$$

To compute the integrals $I_{p, m}$ we use residue theory. We surround the cuts $\Gamma_{n}, n=$ $1, \ldots, N$, by smooth closed curves and shrink these curves to $\Gamma_{n}$. We denote the resulting contour by $\Lambda$. We also construct a circle $C_{r}$ of large radius $r$ centered at the origin.

For example, consider the integral $I_{1, m}$. By passing from the direct value $Q_{1}(s)$ of the function $Q_{1}(z)$ to the limit values $Q_{1}^{ \pm}(s)$ on $\Gamma$ with the use of (41), we obtain

$$
\begin{gathered}
I_{1, m}=\frac{1}{2} \int_{\Gamma}\left(\frac{e^{i \pi \eta}}{Q_{1}(s)}+\frac{e^{-i \pi \eta}}{Q_{1}(s)}\right) s^{m} d s \\
=-\frac{1}{2 i} \int_{\Gamma}\left(\frac{1}{Q_{1}^{+}(s)}-\frac{1}{Q_{1}^{-}(s)}\right) s^{m} d s=-\frac{1}{2 i} \int_{\Lambda} \frac{z^{m}}{Q_{1}(z)} d z .
\end{gathered}
$$

The function $z^{m} / Q_{1}(z)$ is analytic in the domain enclosed between $\Lambda$ and $C_{r}$ and is continuous on the boundary of this domain. By the residue theorem, we have

$$
\int_{\Lambda} \frac{z^{m}}{Q_{1}(z)} d z+\int_{C_{r}} \frac{z^{m}}{Q_{1}(z)} d z=0
$$

for an arbitrary sufficiently large $r$. By letting $r \rightarrow \infty$, we obtain

$$
\begin{aligned}
& I_{1, m}=-\frac{1}{2 i} \int_{\Lambda} \frac{z^{m}}{Q_{1}(z)} d z=\frac{1}{2 i} \lim _{r \rightarrow \infty} \int_{C_{r}} \frac{z^{m}}{Q_{1}(z)} d z \\
& =\pi \lim _{|z| \rightarrow \infty} \frac{|z|^{m+1}}{|z|^{N}}= \begin{cases}0, & m=0, \ldots, N-2, \\
\pi, & m=N-1 .\end{cases}
\end{aligned}
$$

Likewise,

$$
I_{2, m}= \begin{cases}0, & m=0, \ldots, N-2, \\ \pi, & m=N-1 .\end{cases}
$$

We apply the same technique to the Cauchy type integrals $J_{p, m}(s)$ with $s \notin \Gamma$ :

$$
\begin{gathered}
J_{1, m}(s)=-\frac{1}{2 i} \int_{\Gamma}\left(\frac{1}{Q_{1}^{+}(\sigma)}-\frac{1}{Q_{1}^{-}(\sigma)}\right) \sigma^{m} \frac{d \sigma}{\sigma-s} \\
=-\frac{1}{2 i} \int_{\Lambda} \frac{z^{m}}{Q_{1}(z)} \frac{d z}{z-s}=-\frac{1}{2 i} 2 \pi i \operatorname{Res}\left[\frac{z^{m}}{Q_{1}(z)(z-s)}, z=s\right]=-\frac{\pi s^{m}}{Q_{1}(s)}, \quad s \notin \Gamma,
\end{gathered}
$$

where $m=0,1, \ldots, N-1$.

Likewise,

$$
J_{2, m}(s)=-\frac{\pi s^{m}}{Q_{2}(s)}, \quad s \notin \Gamma, \quad m=0,1, \ldots, N-1 .
$$


One can also find the values of the integrals $J_{p, m}(s)$ for $s \in \Gamma$. Note that the function $s^{m}(\cos \pi \eta) / Q_{1}(s)$ is a solution of the homogeneous equation (18) and the function $s^{m}(\sin \pi \eta) / Q_{2}(s)$ is a solution of the homogeneous equation (19). Therefore,

$$
J_{1, m}(s)=\pi \sin \pi \eta \frac{s^{m}}{Q_{1}(s)}, \quad J_{2, m}(s)=-\pi \cos \pi \eta \frac{s^{m}}{Q_{2}(s)}, \quad s \in \Gamma,
$$

where $m=0,1, \ldots, N-1$.

Using this result, we obtain

$$
\int_{\Gamma} \frac{\Phi_{p}(s)}{Q_{p}(s)} d s=0, \quad \int_{\Gamma} \frac{K_{p j}(s, \sigma)}{Q_{p}(s)} d s \equiv 0, \quad \sigma \in \Gamma, \quad p=1,2, j=1,2 .
$$

Let us prove, for example, the first of these equalities. To this end, we represent the function $\Phi_{1}(s)$ from (25) in the form

$$
\Phi_{1}(s)=\operatorname{tg} \pi \eta \widehat{f}_{1}(s) Q_{1}(s)+\frac{1}{\pi} \int_{\Gamma} \frac{\widehat{f}_{1}(\sigma) Q_{1}(\sigma)}{\sigma-s} d \sigma
$$

and straightforwardly verify the following relation by changing the integration order in the double integral:

$$
\int_{\Gamma} \frac{\Phi_{1}(s)}{Q_{1}(s)} d s=\operatorname{tg} \pi \eta \int_{\Gamma} \widehat{f}_{1}(s) d s+\frac{1}{\pi} \int_{\Gamma} d \sigma \widehat{f_{1}}(\sigma) Q_{1}(\sigma)\left(\int_{\Gamma} \frac{1}{Q_{1}(s)} \frac{d s}{\sigma-s}\right) .
$$

By virtue of $(42$,$) , the integral in the round brackets equals -\pi(\operatorname{tg} \pi \eta) / Q_{1}(\sigma)$. Hence, the whole expression equals zero, which proves the assertion.

The remaining relations in (43) can be proved in a similar way.

7. Case of a single cut. Let $N=1$, i.e., let $\Gamma$ contain a single cut:

$$
\Gamma=\left\{x: x=x(s)=\left(x_{1}(s), x_{2}(s)\right), s \in[a, b]\right\} .
$$

In this case, it is possible to simplify Eq. (32). We seek a solution to the problem in the form

$$
u(x)=\frac{1}{2 \sqrt{1+\beta^{2}}}\left(V\left[\rho_{2}-\left(\sqrt{1+\beta^{2}}+\beta\right) \rho_{1}\right](x)+T\left[\rho_{1}+\left(\sqrt{1+\beta^{2}}+\beta\right) \rho_{2}\right](x)\right)+c .
$$


The functions $\rho_{1}(s), \rho_{2}(s) \in C_{q}^{\varpi}(\Gamma)$ and the constant $c$ can be found from the following system of equations:

$$
\begin{gathered}
\frac{1}{2 \sqrt{1+\beta^{2}}}\left(V\left[\rho_{2}-\left(\sqrt{1+\beta^{2}}+\beta\right) \rho_{1}\right](x(a))+T\left[\rho_{1}+\left(\sqrt{1+\beta^{2}}+\beta\right) \rho_{2}\right](x(a))\right) \\
+c=f^{+}(a) .
\end{gathered}
$$

This system follows from the system (18)-(22) if $N=1$. The functions $F_{1}(s)$ and $F_{2}(s)$ are given by (18) and (19). We solve Eq. (45) for $\rho_{1}(s)$ and (46) for $\rho_{2}(s)$ using Lemma 2 and assuming that $F_{1}(s), F_{2}(s)$ are given functions. We have

$$
\rho_{1}(s)=\frac{\left(\sqrt{1+\beta^{2}}-\beta\right)}{2 \sqrt{1+\beta^{2}}} F_{1}(s)+\frac{1}{2 \pi \sqrt{1+\beta^{2}} Q_{1}(s)} \int_{\Gamma} \frac{F_{1}(\sigma) Q_{1}(\sigma)}{\sigma-s} d \sigma-\frac{B_{1} \cos \pi \eta}{Q_{1}(s)},
$$

$$
\rho_{2}(s)=\frac{\left(\sqrt{1+\beta^{2}}+\beta\right)}{2 \sqrt{1+\beta^{2}}} F_{2}(s)-\frac{1}{2 \pi \sqrt{1+\beta^{2}} Q_{2}(s)} \int_{\Gamma} \frac{F_{2}(\sigma) Q_{2}(\sigma)}{\sigma-s} d \sigma-\frac{B_{2} \sin \pi \eta}{Q_{2}(s)},
$$

where $s \in \Gamma ; B_{1}, B_{2}$ are arbitrary real constants,

$$
Q_{1}(s)=(s-a)^{1-\eta}(b-s)^{\eta}, \quad Q_{2}(s)=(s-a)^{1 / 2-\eta}(b-s)^{1 / 2+\eta}, \quad s \in \Gamma,
$$

$Q_{1}(s), Q_{2}(s) \in C^{0, \eta_{0}}(\Gamma)$, the constant $\eta$ is defined in Lemma $2, \eta_{0}=\min \{\eta, 1 / 2-\eta\}$. By substituting functions $\rho_{1}(s), \rho_{2}(s)$ from (50) and (51) into (47), (48) and by using formulas from Section 6 , we arrive at the system of linear algebraic equations for $B_{1}, B_{2}$ :

$$
\begin{gathered}
B_{1}+\left(\sqrt{1+\beta^{2}}+\beta\right) B_{2}=0, \\
\left(\sqrt{1+\beta^{2}}+\beta\right) B_{1}-B_{2}=-4 A \sqrt{1+\beta^{2}} .
\end{gathered}
$$

The solution is

$$
B_{1}=-4 A \sqrt{1+\beta^{2}} \sin \pi \eta \cos \pi \eta, \quad B_{2}=4 A \sqrt{1+\beta^{2}} \sin ^{2} \pi \eta .
$$


Writing out the expressions for $F_{1}(s), F_{2}(s)$, we obtain the system of regularized equations for $\rho_{1}(s), \rho_{2}(s)$ :

$$
\begin{aligned}
& \rho_{1}(s)+\frac{1}{Q_{1}(s)} \int_{\Gamma} \rho_{1}(\sigma) K_{11}(s, \sigma) d \sigma+\frac{1}{Q_{1}(s)} \int_{\Gamma} \rho_{2}(\sigma) K_{12}(s, \sigma) d \sigma=\frac{\Phi_{1}(s)}{Q_{1}(s)}, \\
& \rho_{2}(s)+\frac{1}{Q_{2}(s)} \int_{\Gamma} \rho_{1}(\sigma) K_{21}(s, \sigma) d \sigma+\frac{1}{Q_{2}(s)} \int_{\Gamma} \rho_{2}(\sigma) K_{22}(s, \sigma) d \sigma=\frac{\Phi_{2}(s)}{Q_{2}(s)},
\end{aligned}
$$

here $s \in \Gamma$;

$$
\begin{gathered}
\Phi_{1}(s)=\left(\sqrt{1+\beta^{2}}-\beta\right)\left(f^{\prime}+(s)+\frac{f^{-}(s)}{\sqrt{1+\beta^{2}}}\right) Q_{1}(s) \\
+\frac{1}{\pi} \int_{\Gamma} \frac{Q_{1}(\sigma)}{\sigma-s}\left(f^{\prime}+(\sigma)+\frac{f^{-}(\sigma)}{\sqrt{1+\beta^{2}}}\right) d \sigma+4 A \sqrt{1+\beta^{2}} \sin \pi \eta \cos ^{2} \pi \eta \\
\Phi_{2}(s)=\left(f^{\prime}+(s)-\frac{f^{-}(s)}{\sqrt{1+\beta^{2}}}\right) Q_{2}(s) \\
-\frac{\left(\sqrt{1+\beta^{2}}-\beta\right)}{\pi} \int_{\Gamma} \frac{Q_{2}(\sigma)}{\sigma-s}\left(f^{\prime}+(\sigma)-\frac{f^{-}(\sigma)}{\sqrt{1+\beta^{2}}}\right) d \sigma-4 A \sqrt{1+\beta^{2}} \sin ^{3} \pi \eta .
\end{gathered}
$$

Let us transform Eqs. (153) and (54) in the same way as Eqs. (23) and (24). Namely, we introduce functions $\rho_{j *}(s)=Q_{j}(s) \rho_{j}(s), j=1,2$, and operators $\mathbf{K}_{p j}, p=1,2, j=1,2$ (see (30)). As a result, Eqs. (53) and (54) are reduced to a vector equation for an unknown vector column $\bar{\rho}=\left(\rho_{1 *}(s), \rho_{2 *}(s)\right)^{T}$ from Banach space $C^{0}(\Gamma) \times C^{0}(\Gamma)$ with the norm $\|\bar{\rho}\|_{C^{0}(\Gamma) \times C^{0}(\Gamma)}=\left\|\rho_{1 *}\right\|_{C^{0}(\Gamma)}+\left\|\rho_{2 *}\right\|_{C^{0}(\Gamma)}$; that is,

$$
(\mathbf{I}+\mathbf{R}) \bar{\rho}=\bar{\Phi}, \quad \mathbf{R}=\left(\begin{array}{ll}
\mathbf{K}_{11} & \mathbf{K}_{12} \\
\mathbf{K}_{21} & \mathbf{K}_{22}
\end{array}\right)
$$

where $\bar{\Phi}=\left(\Phi_{1}(s), \Phi_{2}(s)\right)^{T} \in C^{0}(\Gamma) \times C^{0}(\Gamma)$, the functions $\Phi_{1}(s), \Phi_{2}(s)$ are given by (55) and (56), $\mathbf{I}$ is the identity matrix mapping the space $C^{0}(\Gamma) \times C^{0}(\Gamma)$ into itself, the operators $\mathbf{K}_{p j}(p=1,2, j=1,2)$ are defined by (30).

Obviously, (57) is a Fredholm equation in the space $C^{0}(\Gamma) \times C^{0}(\Gamma)$ and it has a unique solution in this space. Furthermore, if $\Phi_{1}(s)$ and $\Phi_{2}(s)$ are defined by (55) and (56), then the solution of Eq. (57) from $C^{0}(\Gamma) \times C^{0}(\Gamma)$ belongs to $C^{0, \omega}(\Gamma) \times C^{0, \omega}(\Gamma)$ with $\omega=\min \{\lambda, \eta, 1 / 2-\eta\}$. The proof is similar to that for Eq. (32).

Let $\bar{\rho}=\left(\rho_{1 *}(s), \rho_{2 *}(s)\right)^{T}$ be a solution of Eq. (57) in the space $C^{0, \omega}(\Gamma) \times C^{0, \omega}(\Gamma)$. Then the solution of Problem $\mathcal{S}$ with the single cut is given by (44), where $\rho_{j}(s)=$ 
$\rho_{j *}(s) Q_{j}^{-1}(s), j=1,2$, and the constant $c$ is uniquely defined from the condition (49):

$$
\begin{aligned}
c= & f^{+}(a)-\frac{1}{2 \sqrt{1+\beta^{2}}} V\left[\frac{\rho_{2 *}}{Q_{2}}-\left(\sqrt{1+\beta^{2}}+\beta\right) \frac{\rho_{1 *}}{Q_{1}}\right](x(a)) \\
& -\frac{1}{2 \sqrt{1+\beta^{2}}} T\left[\frac{\rho_{1 *}}{Q_{1}}+\left(\sqrt{1+\beta^{2}}+\beta\right) \frac{\rho_{2 *}}{2 Q_{2}}\right](x(a)) .
\end{aligned}
$$

8. Behavior of the solution gradient at the end of the cut. Let $u$ be the solution of Problem $\mathcal{S}$ with the single cut obtained in Section 7. Using the notation introduced above, we investigate the behavior of $\nabla u$ at the ends of $\Gamma=\{x: x=x(s)=$ $\left.\left(x_{1}(s), x_{2}(s)\right), s \in[a, b]\right\}$. Let $x(d)$ be one of these endpoints. In a neighborhood of $x(d)$ we introduce the system of polar coordinates

$$
x_{1}=|x-x(d)| \cos \varphi, \quad x_{2}=|x-x(d)| \sin \varphi .
$$

We recall that $\alpha(s)$ is the angle between the direction of $O x_{1}$ axis and the tangent vector $\tau_{x}$ at the point $x(s) \in \Gamma$. We assume that $\varphi \in(\alpha(d), \alpha(d)+2 \pi)$ if $d=a$ and $\varphi \in(\alpha(d)-\pi, \alpha(d)+\pi)$ if $d=b$. We put $\alpha(a)=\alpha(a+0), \alpha(b)=\alpha(b-0)$. Thus, the angle $\varphi$ varies continuously in a neighborhood of the point $x(d)$ cut along $\Gamma$.

Let us represent the singularities of the functions $\mu(s)$ and $\nu(s)$ in the explicit form:

(1) at the end $a$ :

$$
\begin{aligned}
& \mu(s)=\frac{1}{2 \sqrt{1+\beta^{2}}}\left(\frac{\rho_{2}^{a}(s)}{|s-a|^{1-\eta}}-\left(\sqrt{1+\beta^{2}}+\beta\right) \frac{\rho_{1}^{a}(s)}{|s-a|^{1 / 2-\eta}}\right), \\
& \nu(s)=\frac{1}{2 \sqrt{1+\beta^{2}}}\left(\left(\sqrt{1+\beta^{2}}+\beta\right) \frac{\rho_{2}^{a}(s)}{|s-a|^{1-\eta}}+\frac{\rho_{1}^{a}(s)}{|s-a|^{1 / 2-\eta}}\right) ;
\end{aligned}
$$

(2) at the end $b$ :

$$
\begin{aligned}
& \mu(s)=\frac{1}{2 \sqrt{1+\beta^{2}}}\left(\frac{\rho_{2}^{b}(s)}{|s-b|^{\eta}}-\left(\sqrt{1+\beta^{2}}+\beta\right) \frac{\rho_{1}^{b}(s)}{|s-b|^{1 / 2+\eta}}\right), \\
& \nu(s)=\frac{1}{2 \sqrt{1+\beta^{2}}}\left(\left(\sqrt{1+\beta^{2}}+\beta\right) \frac{\rho_{2}^{b}(s)}{|s-b|^{\eta}}+\frac{\rho_{1}^{b}(s)}{|s-b|^{1 / 2+\eta}}\right) .
\end{aligned}
$$

Here

$$
\rho_{1}^{a}(s)=\frac{\rho_{1 *}(s)}{|s-b|^{1 / 2+\eta}}=\rho_{1}(s)|s-a|^{1 / 2-\eta}, \quad \rho_{2}^{a}(s)=\frac{\rho_{2 *}(s)}{|s-b|^{\eta}}=\rho_{2}(s)|s-a|^{1-\eta}
$$

are Hölder functions on $\Gamma$ in a neighborhood of $a$ and

$$
\rho_{1}^{b}(s)=\frac{\rho_{1 *}(s)}{|s-a|^{1 / 2-\eta}}=\rho_{1}(s)|s-b|^{1 / 2+\eta}, \quad \rho_{2}^{b}(s)=\frac{\rho_{2 *}(s)}{|s-a|^{1-\eta}}=\rho_{2}(s)|s-b|^{\eta}
$$

are Hölder functions on $\Gamma$ in a neighborhood of $b$. The functions $\rho_{1 *}(s), \rho_{2 *}(s)$ are the components of the solution of Eq. (57). 
Using the results [6] on the behavior of Cauchy type integrals at the ends of $\Gamma$, we arrive at the following assertion.

Theorem 5. Let $x \rightarrow x(d) \in X$, where $d=a$ or $d=b$; then in a neighborhood of $x(d)$ the derivatives of the solution of Problem $\mathcal{S}$ can be expressed by the following formulas:

(1) if $d=a$,

$$
\begin{aligned}
& \left.\frac{\partial u}{\partial x_{1}}\right|_{x \rightarrow x(a)}=-\frac{\rho_{1}^{a}(a) \sin \vartheta^{a}(1 / 2-\eta)}{2|x-x(a)|^{1 / 2-\eta}}+\left(\sqrt{1+\beta^{2}}+\beta\right) \frac{\rho_{2}^{a}(a) \sin \vartheta^{a}(1-\eta)}{2|x-x(a)|^{1-\eta}}+O(1), \\
& \left.\frac{\partial u}{\partial x_{2}}\right|_{x \rightarrow x(a)}=\frac{\rho_{1}^{a}(a) \cos \vartheta^{a}(1 / 2-\eta)}{2|x-x(a)|^{1 / 2-\eta}}-\left(\sqrt{1+\beta^{2}}+\beta\right) \frac{\rho_{2}^{a}(a) \cos \vartheta^{a}(1-\eta)}{2|x-x(a)|^{1-\eta}}+O(1)
\end{aligned}
$$

(2) if $d=b$,

$$
\begin{aligned}
& \left.\frac{\partial u}{\partial x_{1}}\right|_{x \rightarrow x(b)}=\frac{\rho_{1}^{b}(b) \cos \vartheta^{b}(1 / 2+\eta)}{2|x-x(b)|^{1 / 2+\eta}}+\left(\sqrt{1+\beta^{2}}+\beta\right) \frac{\rho_{2}^{b}(b) \sin \vartheta^{b}(\eta)}{2|x-x(b)|^{\eta}}+O(1), \\
& \left.\frac{\partial u}{\partial x_{2}}\right|_{x \rightarrow x(b)}=\frac{\rho_{1}^{b}(b) \sin \vartheta^{b}(1 / 2+\eta)}{2|x-x(b)|^{1 / 2+\eta}}-\left(\sqrt{1+\beta^{2}}+\beta\right) \frac{\rho_{2}^{b}(b) \cos \vartheta^{b}(\eta)}{2|x-x(b)|^{\eta}}+O(1) .
\end{aligned}
$$

Here,

$$
\vartheta^{a}(\gamma)=\gamma \varphi+(1-\gamma) \alpha(a), \quad \vartheta^{b}(\gamma)=\gamma \varphi+(1-\gamma) \alpha(b)-\pi \eta,
$$

functions denoted as $O(1)$ are continuous both at the point $x(d)$ and in a neighborhood of the point $x(d)$ cut along $\Gamma$. The functions $\rho_{1}^{d}(s)$ and $\rho_{2}^{d}(s)(d=a, b)$ are introduced in (61) and (62).

Proof. We introduce a complex coordinate $\widetilde{x}$ expressed via the Cartesian coordinates $x=\left(x_{1}, x_{2}\right)$ by the relation $\widetilde{x}=x_{1}+i x_{2}$. We write out the representation of the derivatives of the angular and logarithmic potentials in terms of the Cauchy type integral in the complex plane [4:

$$
\begin{gathered}
\frac{\partial V[\mu](x)}{\partial x_{1}}=-\operatorname{Im} \Omega[\mu](\widetilde{x}), \quad \frac{\partial V[\mu](x)}{\partial x_{2}}=-\operatorname{Re} \Omega[\mu](\widetilde{x}), \\
\frac{\partial T[\nu](x)}{\partial x_{1}}=\operatorname{Re} \Omega[\nu](\widetilde{x}), \quad \frac{\partial T[\nu](x)}{\partial x_{2}}=-\operatorname{Im} \Omega[\nu](\widetilde{x}) .
\end{gathered}
$$

Here

$$
\Omega[\mu](\widetilde{x})=\frac{1}{2 \pi i} \int_{\Gamma} \mu(s) e^{-i \alpha(s)} \frac{d \widetilde{y}(s)}{\widetilde{y}(s)-\widetilde{x}}, \quad \widetilde{y}=y_{1}+i y_{2} .
$$

It follows from (44) and (63) that the investigation of the behavior of $\nabla u$ can be reduced to the study of the behavior of the Cauchy type integrals

$$
\begin{gathered}
\Omega\left[\rho_{1}^{a}(s) /|s-a|^{1 / 2-\eta}\right](\widetilde{x}), \quad \Omega\left[\rho_{2}^{a}(s) /|s-a|^{1-\eta}\right](\widetilde{x}), \\
\Omega\left[\rho_{1}^{b}(s) /|s-b|^{1 / 2+\eta}\right](\widetilde{x}), \quad \Omega\left[\rho_{2}^{b}(s) /|s-b|^{\eta}\right](\widetilde{x})
\end{gathered}
$$

in the complex plane near the ends of $\Gamma$. 
We give a detailed investigation for the fourth of these functions. (The others can be treated in a similar way.) We have

$$
\Omega\left[\frac{\rho_{2}^{b}(s)}{|s-b|^{\eta}}\right](\widetilde{x})=\Omega\left[\Psi_{2}^{b}(s)\right](\widetilde{x})+\rho_{2}^{b}(b) \Omega\left[\frac{1}{|s-b|^{\eta}}\right](\widetilde{x})
$$

where $\Psi_{2}^{b}(s)=\left(\rho_{2}^{b}(s)-\rho_{2}^{b}(b)\right) /|s-b|^{\eta}$.

Let $d=a$ or $d=b$. Let us transform the density of the integral $\Omega\left[1 /|s-d|^{\gamma}\right](\widetilde{x})$ with $\gamma \in(0,1)$ :

$$
\frac{e^{-i \alpha(s)}}{|s-d|^{\gamma}}=\frac{\widetilde{\phi}(\widetilde{y}(s))}{(\widetilde{y}(s)-\widetilde{x}(d))^{\gamma}}
$$

where

$$
\begin{aligned}
& \widetilde{\phi}(\widetilde{y}(s))=\phi(s)=e^{-i \alpha(s)}\left[\left(\frac{\widetilde{y}(s)-\widetilde{x}(d)}{|s-d|}\right)^{\gamma}\right] \\
= & e^{-i \alpha(s)}\left|\frac{\widetilde{y}(s)-\widetilde{x}(d)}{s-d}\right|^{\gamma} \exp \{i \gamma \arg (\widetilde{y}(s)-\widetilde{x}(d))\} .
\end{aligned}
$$

Using the technique from [4, Lemma 1], one can readily prove that the function $\widetilde{g}(s)=$ $(\widetilde{y}(s)-\widetilde{x}(d)) /|s-d|$ belongs to the class $C^{1}(\Gamma)$ and does not vanish for any $s \in \Gamma$. Note that $(\widetilde{g})^{\gamma}$ is a Hölder function with the exponent 1 on an arbitrary smooth arc which does not contain the point $\widetilde{g}=0$. Using the theory of composite functions, one can readily prove that the expression in the square brackets in (65) is a Hölder function of $s$ on $\Gamma$ in a neighborhood of $d$ with the exponent 1. Obviously, $e^{-i \alpha(s)}=\left(y_{1}^{\prime}(s)-i y_{2}^{\prime}(s)\right) \in C^{1}(\Gamma)$. Hence, $\phi(s)$ is a Hölder function with respect to $s$ on $\Gamma$ in a neighborhood of $d$ with the exponent 1; i.e., for arbitrary points $s_{1}$ and $s_{2}$ lying on $\Gamma$ in a neighborhood of $d$, we have

$$
\left|\widetilde{\phi}\left(\widetilde{y}\left(s_{2}\right)\right)-\widetilde{\phi}\left(\widetilde{y}\left(s_{1}\right)\right)\right|=\left|\phi\left(s_{2}\right)-\phi\left(s_{1}\right)\right| \leq \mathrm{const}\left|s_{2}-s_{1}\right| .
$$

Let us prove that $\widetilde{\phi}(\widetilde{y}(s))=\phi(s)$ is a Hölder function of $\widetilde{y}$ on $\Gamma$ in a neighborhood of $\widetilde{x}(d)$. By [4, Lemma 1], the function $\left|s_{2}-s_{1}\right| /\left|\widetilde{y}\left(s_{2}\right)-\widetilde{y}\left(s_{1}\right)\right|$ belongs to $C^{1}(\Gamma \times \Gamma) \subset C^{0}(\Gamma \times \Gamma)$ with respect to both variables $s_{1}, s_{2}$ and is therefore uniformly bounded with respect to $s_{1}, s_{2} \in \Gamma$ :

$$
\frac{\left|s_{2}-s_{1}\right|}{\left|\widetilde{y}\left(s_{2}\right)-\widetilde{y}\left(s_{1}\right)\right|} \leq c^{*} .
$$

Hence, $\left|s_{2}-s_{1}\right| \leq c^{*}\left|\widetilde{y}\left(s_{2}\right)-\widetilde{y}\left(s_{1}\right)\right|$. Using this estimate in (66), we have

$$
\left|\widetilde{\phi}\left(\widetilde{y}_{2}\right)-\widetilde{\phi}\left(\widetilde{y}_{1}\right)\right| \leq c_{0}\left|\widetilde{y}_{2}-\widetilde{y}_{1}\right|,
$$

where $\widetilde{y}_{2}=\widetilde{y}\left(s_{2}\right), \widetilde{y}_{1}=\widetilde{y}\left(s_{1}\right)$ are arbitrary points lying on $\Gamma$ in a neighborhood of $\widetilde{x}(d)$, and $c_{0}$ is a constant. Therefore, $\widetilde{\phi}(\widetilde{y})$ is a Hölder function of $\widetilde{y}$ on $\Gamma$ in a neighborhood of $\widetilde{x}(d)$ with the exponent 1 . 
We transform the integral $\Omega\left[1 /|s-d|^{\gamma}\right](\widetilde{x})$ as follows:

$$
\begin{gathered}
\Omega\left[\frac{1}{|s-d|^{\gamma}}\right](\widetilde{x})=\frac{1}{2 \pi i} \int_{\Gamma} \frac{\widetilde{\phi}(\widetilde{y})}{(\widetilde{y}-\widetilde{x}(d))^{\gamma}} \frac{d \widetilde{y}-\widetilde{x}}{\widetilde{y}} \\
=\frac{\widetilde{\phi}(\widetilde{x}(d))}{2 \pi i} \int_{\Gamma} \frac{1}{(\widetilde{y}-\widetilde{x}(d))^{\gamma}} \frac{d \widetilde{y}-\widetilde{x}}{\widetilde{y}}+\frac{1}{2 \pi i} \int_{\Gamma} \frac{\widetilde{\phi}(\widetilde{y})-\widetilde{\phi}(\widetilde{x}(d))}{(\widetilde{y}-\widetilde{x}(d))^{\gamma}} \frac{d \widetilde{y}}{\widetilde{y}-\widetilde{x}}
\end{gathered}
$$

the function $\widetilde{\phi}(\widetilde{y})$ has been introduced in (65). By virtue of the estimate (68) and results of [6. Sec. 22], the second term in the latter formula is a function $O(1)$ continuous in a neighborhood of the point $x(d)$ cut along $\Gamma$. We apply the results of [6] to the first term. The behavior of the Cauchy type integral

$$
I(\widetilde{x})=\frac{1}{2 \pi i} \int_{\Gamma} \frac{1}{(\widetilde{y}-\widetilde{x}(d))^{\gamma}} \frac{d \widetilde{y}}{\widetilde{y}-\widetilde{x}}
$$

where $\gamma \in(0,1)$, was studied in [6, Sec. 23]. For $\widetilde{x}$ which are close enough to $\widetilde{x}(d)$, but do not lie on $\Gamma$, the representation [6, Sec. 23]

$$
I(\widetilde{x})=(-1)^{j(d)} \frac{\exp \left\{(-1)^{j(d)} \gamma \pi i\right\}}{2 i \sin \gamma \pi} \frac{1}{(\widetilde{x}-\widetilde{x}(d))^{\gamma}}+O(1)
$$

is valid, where $(\widetilde{x}-\widetilde{x}(d))^{\gamma}$ denotes a branch, which is holomorphic near $\widetilde{x}(d)$ in a plane cut along $\Gamma$ and equals $(\widetilde{x}(s)-\widetilde{x}(d))^{\gamma}$ on the left side of the cut; $O(1)$ is introduced in Theorem $5 ; j(a)=2, j(b)=1$.

$$
\begin{aligned}
& \text { If } s \in \Gamma \text { and } s \rightarrow d \text {, then }|\widetilde{y}(s)-\widetilde{x}(d)| /|s-d| \rightarrow 1 \text { and } \\
& \qquad \arg (\widetilde{y}(s)-\widetilde{x}(d)) \rightarrow \alpha(d)+\pi(2-j(d)) .
\end{aligned}
$$

Taking into account this relation, we compute the limit values of the function $\widetilde{\phi}(\widetilde{y})$ from (65) as $\widetilde{y} \rightarrow \widetilde{x}(d)$ and $\widetilde{y} \in \Gamma$. Using (69), we obtain

$$
\begin{gathered}
\Omega\left[\frac{1}{|s-d|^{\gamma}}\right](\widetilde{x})=(-1)^{j(d)} \frac{\exp \left\{(-1)^{j(d)} \gamma \pi i\right\}}{2 i \sin \gamma \pi} \frac{1}{(\widetilde{x}-\widetilde{x}(d))^{\gamma}} \\
\times \exp \{i(-\alpha(d)+\gamma(\alpha(d)+\pi(2-j(d))))\}+O(1) \\
=\frac{1}{2 \sin \gamma \pi} \frac{1}{|x-x(d)|^{\gamma}} \exp \left(i \theta_{d}(\gamma)\right)+O(1),
\end{gathered}
$$

where $\theta_{a}(\gamma)=-\gamma \varphi-(1-\gamma) \alpha(a)+(\gamma-1 / 2) \pi$ and $\theta_{b}(\gamma)=-\gamma \varphi-(1-\gamma) \alpha(b)+\pi / 2$.

Now we investigate the behavior of the function $\Omega\left[\Psi_{2}^{b}(s)\right](\widetilde{x})$ in (64). To this end, we study the function $\Psi_{2}^{b}(s)$. Note that the function $\rho_{2}(s)=\rho_{2}^{b}(s) /|s-b|^{\eta}$ satisfies Eq. (51). Using this equation and results of [6, Sec. 22], one can prove the following assertion.

Lemma 8. If $F_{2}(s)$ from (51) is a Hölder function on $\Gamma$, then $\Psi_{2}^{b}(s)$ is also a Hölder function on $\Gamma$ near $b$. In addition, $\Psi_{2}^{b}(b)=\lim _{s \rightarrow b} \Psi_{2}^{b}(s)=0$.

The proof of the lemma is given in the next section. It follows from Lemma 8 and estimate (67) that $\widetilde{\Psi}_{2}^{b}(\widetilde{y}(s))=\Psi_{2}^{b}(s)$ considered as a function of the complex variable $\widetilde{y}$, is a Hölder function on $\Gamma$ near $\widetilde{x}(b)$. Besides, $\widetilde{\Psi}_{2}^{b}(\widetilde{x}(b))=0$. Hence, by [6, Sec. 22], the 
function $\Omega\left[\Psi_{2}^{b}(s)\right](\widetilde{x})$ is continuously extendible to the end $\widetilde{x}(b)$. Using the notation $O(1)$ introduced in Theorem 5, we have $\Omega\left[\Psi_{2}^{b}(s)\right]=O(1)$ as $\widetilde{x} \rightarrow \widetilde{x}(b)$.

Returning to the original integral (64) and using (71), we obtain

$$
\Omega\left[\frac{\rho_{2}^{b}(s)}{|s-b|^{\eta}}\right](\widetilde{x})=\frac{\rho_{2}^{b}(b)}{2 \sin \pi \eta} \frac{1}{|x-x(b)|^{\eta}} e^{i \theta_{b}(\eta)}+O(1)
$$

as $\widetilde{x} \rightarrow \widetilde{x}(b)$.

In a similar way, one can show that, as $\widetilde{x} \rightarrow \widetilde{x}(d)$, the formula

$$
\Omega\left[\frac{\rho_{j}^{d}(s)}{|s-d|^{\gamma}}\right](\widetilde{x})=\frac{\rho_{j}^{d}(d)}{2 \sin \pi \gamma} \frac{1}{|x-x(d)|^{\gamma}} e^{i \theta_{d}(\gamma)}+O(1)
$$

is valid, where $d=a, j=2, \gamma=1-\eta$ or $d=a, j=1, \gamma=1 / 2-\eta$, or $d=b, j=$ $1, \gamma=1 / 2+\eta$.

Using formulas (59), (71), (72), we obtain the following relations as $\widetilde{x} \rightarrow \widetilde{x}(d)$ :

(1) if $d=a$,

$$
\begin{aligned}
& \Omega[\mu](\widetilde{x})=\frac{1}{2 \sqrt{1+\beta^{2}}}\left(\frac{\rho_{2}^{a}(a)}{|x-x(a)|^{1-\eta}} \frac{e^{i \theta_{a}(1-\eta)}}{2 \sin \pi \eta}-\frac{\rho_{1}^{a}(a)}{|x-x(a)|^{1 / 2-\eta}} \frac{e^{i \theta_{a}(1 / 2-\eta)}}{2 \sin \pi \eta}\right)+O(1), \\
& \Omega[\nu](\widetilde{x})=\frac{1}{2 \sqrt{1+\beta^{2}}}\left(\frac{\rho_{2}^{a}(a) e^{i \theta_{a}(1-\eta)}}{|x-x(a)|^{1-\eta}} \frac{\cos \pi \eta}{2 \sin ^{2} \pi \eta}+\frac{\rho_{1}^{a}(a)}{|x-x(a)|^{1 / 2-\eta}} \frac{e^{i \theta_{a}(1 / 2-\eta)}}{2 \cos \pi \eta}\right)+O(1)
\end{aligned}
$$

(2) if $d=b$,

$$
\begin{gathered}
\Omega[\mu](\widetilde{x})=\frac{1}{2 \sqrt{1+\beta^{2}}}\left(\frac{\rho_{2}^{b}(b)}{|x-x(b)|^{\eta}} \frac{e^{i \theta_{b}(\eta)}}{2 \sin \pi \eta}-\frac{\rho_{1}^{b}(b)}{|x-x(b)|^{1 / 2+\eta}} \frac{e^{i \theta_{b}(1 / 2+\eta)}}{2 \sin \pi \eta}\right)+O(1), \\
\Omega[\nu](\widetilde{x})=\frac{1}{2 \sqrt{1+\beta^{2}}}\left(\frac{\rho_{2}^{b}(b)}{|x-x(b)|^{\eta}} \frac{e^{i \theta_{b}(\eta)} \cos \pi \eta}{2 \sin ^{2} \pi \eta}+\frac{\rho_{1}^{b}(b)}{|x-x(b)|^{1 / 2+\eta}} \frac{e^{i \theta_{b}(1 / 2+\eta)}}{2 \cos \pi \eta}\right)+O(1) .
\end{gathered}
$$

Using (44) and (63), as $\widetilde{x} \rightarrow \widetilde{x}(d)$, we have:

(1) if $d=a$,

$$
\begin{aligned}
& \left.\frac{\partial u}{\partial x_{1}}\right|_{x \rightarrow x(a)}=\frac{\rho_{1}^{a}(a)}{2 \sqrt{1+\beta^{2}}|x-x(a)|^{1 / 2-\eta}}\left\{\frac{\sin \theta_{a}(1 / 2-\eta)}{2 \sin \pi \eta}+\frac{\cos \theta_{a}(1 / 2-\eta)}{2 \cos \pi \eta}\right\} \\
& +\frac{\rho_{2}^{a}(a)}{2 \sqrt{1+\beta^{2}}|x-x(a)|^{1-\eta}}\left\{-\frac{\sin \theta_{a}(1-\eta)}{2 \sin \pi \eta}+\frac{\cos \pi \eta \cos \theta_{a}(1-\eta)}{2 \sin ^{2} \pi \eta}\right\}+O(1), \\
& \left.\frac{\partial u}{\partial x_{2}}\right|_{x \rightarrow x(a)}=\frac{\rho_{1}^{a}(a)}{2 \sqrt{1+\beta^{2}}|x-x(a)|^{1 / 2-\eta}}\left\{\frac{\cos \theta_{a}(1 / 2-\eta)}{2 \sin \pi \eta}-\frac{\sin \theta_{a}(1 / 2-\eta)}{2 \cos \pi \eta}\right\} \\
& +\frac{\rho_{2}^{a}(a)}{2 \sqrt{1+\beta^{2}}|x-x(a)|^{1-\eta}}\left\{-\frac{\cos \theta_{a}(1-\eta)}{2 \sin \pi \eta}-\frac{\cos \pi \eta \sin \theta_{a}(1-\eta)}{2 \sin ^{2} \pi \eta}\right\}+O(1) ;
\end{aligned}
$$


2) if $d=b$,

$$
\begin{gathered}
\left.\frac{\partial u}{\partial x_{1}}\right|_{x \rightarrow x(b)}=\frac{\rho_{1}^{b}(b)}{2 \sqrt{1+\beta^{2}}|x-x(b)|^{1 / 2+\eta}}\left\{\frac{\sin \theta_{b}(1 / 2+\eta)}{2 \sin \pi \eta}+\frac{\cos \theta_{b}(1 / 2+\eta)}{2 \cos \pi \eta}\right\} \\
+\frac{\rho_{2}^{b}(b)}{2 \sqrt{1+\beta^{2}}|x-x(b)|^{\eta}}\left\{-\frac{\sin \theta_{b}(\eta)}{2 \sin \pi \eta}+\frac{\cos \theta_{b}(\eta) \cos \pi \eta}{2 \sin ^{2} \pi \eta}\right\}+O(1) \\
\left.\frac{\partial u}{\partial x_{2}}\right|_{x \rightarrow x(b)}=\frac{\rho_{1}^{b}(b)}{2 \sqrt{1+\beta^{2}}|x-x(b)|^{1 / 2+\eta}}\left\{\frac{\cos \theta_{b}(1 / 2+\eta)}{2 \sin \pi \eta}-\frac{\sin \theta_{b}(1 / 2+\eta)}{2 \cos \pi \eta}\right\} \\
+\frac{\rho_{2}^{b}(b)}{2 \sqrt{1+\beta^{2}}|x-x(b)|^{\eta}}\left\{-\frac{\cos \theta_{b}(\eta)}{2 \sin \pi \eta}-\frac{\cos \pi \eta \sin \theta_{b}(\eta)}{2 \sin ^{2} \pi \eta}\right\}+O(1) .
\end{gathered}
$$

Elementary trigonometric transformations complete the proof of the theorem.

Theorem 5 shows that, as a rule, the partial derivatives of a solution of Problem $\mathcal{S}$ have power-law singularities at the ends of the cut with the exponent $q=\max \{1 / 2+\eta, 1-\eta\}$.

9. Proof of Lemma 8. Let us prove Lemma 8 used in Theorem 5 .

We recall that $\Psi_{2}^{b}(s)=\left(\rho_{2}^{b}(s)-\rho_{2}^{b}(b)\right) /|s-b|^{\eta}$ and $\rho_{2}^{b}(s)=\rho_{2}(s)|s-b|^{\eta}$. As was mentioned above, the function $\rho_{2}(s)$ satisfies Eq. (51). Hence, for $\rho_{2}^{b}(s)$ the identity

$$
\rho_{2}^{b}(s)=\mathcal{F}_{2}(s)|s-b|^{\eta}-\frac{1}{|s-a|^{1-\eta}} \int_{\Gamma} \frac{Q_{2}(\xi) \widetilde{\mathcal{F}}_{2}(\xi)}{\xi-s} d \xi-\frac{B_{2} \sin \pi \eta}{|s-a|^{1-\eta}}, \quad s \in \Gamma,
$$

is valid, where $\mathcal{F}_{2}(s)=\left(\sqrt{1+\beta^{2}}+\beta\right) F_{2}(s) /\left(2 \sqrt{1+\beta^{2}}\right), \quad \widetilde{\mathcal{F}}_{2}(s)=F_{2}(s) /\left(2 \pi \sqrt{1+\beta^{2}}\right)$; $\mathcal{F}_{2}(s), \widetilde{\mathcal{F}}_{2}(s) \in C^{0, \lambda}(\Gamma)$. Note that for $s$ lying on $\Gamma$ in a neighborhood of the point $b$, the equality

$$
\frac{1}{|s-a|^{1-\eta}}=\frac{1}{|b-a|^{1-\eta}}+O(|s-b|)
$$

is valid, where $O(|s-b|)$ denotes a Hölder function on $\Gamma$ in a neighborhood of the end $b$ with the exponent 1 . We transform the expression (73) as follows:

$$
\rho_{2}^{b}(s)=\mathcal{F}_{2}(s)|s-b|^{\eta}-\frac{1}{(b-a)^{1-\eta}} \int_{\Gamma} \frac{Q_{2}(\xi) \widetilde{\mathcal{F}}_{2}(\xi)}{\xi-s} d \xi-\frac{B_{2} \sin \pi \eta}{(b-a)^{1-\eta}}+O(|s-b|) \Xi(s),
$$

where $\Xi(s)$ is a Hölder function on $\Gamma$ in a neighborhood of the end $b$ with the exponent $\min \{\eta, \lambda\}$. Now we put $s=b$ in (173) and compute a function $\left[\rho_{2}^{b}(s)-\rho_{2}^{b}(b)\right]$, using Eqs. (73) and (74):

$$
\rho_{2}^{b}(s)-\rho_{2}^{b}(b)=\mathcal{F}_{2}(s)|s-b|^{\eta}+\frac{b-s}{(b-a)^{1-\eta}} \int_{\Gamma} \frac{(\xi-a)^{1-\eta} \widetilde{\mathcal{F}}_{2}(\xi)}{(b-\xi)^{1-\eta}(\xi-s)} d \xi+O(|s-b|) \Xi(s) .
$$

Thus, the function $\Psi_{2}^{b}(s)$ has the form

$$
\Psi_{2}^{b}(s)=\mathcal{F}_{2}(s)-\frac{(b-s)^{1-\eta}}{(b-a)^{1-\eta}} \int_{\Gamma} \frac{(\xi-a)^{1-\eta} \widetilde{\mathcal{F}}_{2}(\xi)}{(b-\xi)^{1-\eta}(\xi-s)} d \xi+O\left(|s-b|^{1-\eta}\right) \Xi(s) .
$$


Using the results of [6, Sec. 22, item 4] and the fact that $\mathcal{F}_{2}(s)$ and $\widetilde{\mathcal{F}}_{2}(s)$ are Hölder functions on $\Gamma$, one can state that $\Psi_{2}^{b}(s)$ is a Hölder function on $\Gamma$ near $b$. Note $[6$, Sec. 22, items 2, 4] that for a function $f(\xi)$ satisfying the Hölder condition on $\Gamma$ in a neighborhood of the end $d$, the formula

$$
\lim _{s \rightarrow d}\left(\frac{(s-d)^{\gamma}}{2 \pi} \int_{\Gamma} \frac{f(\xi)}{(\xi-d)^{\gamma}(\xi-s)} d \xi\right)=\frac{(-1)^{j(d)} \operatorname{ctg} \pi \gamma}{2} f(d)
$$

is valid, where $0<\gamma<1, j(a)=2, j(b)=1$. Applying this formula to the expression $\Psi_{2}^{b}(b)=\lim _{s \rightarrow b} \Psi_{2}^{b}(s)$, we obtain

$$
\begin{aligned}
\Psi_{2}^{b}(b) & =\mathcal{F}_{2}(b)+\frac{2 \pi}{2(b-a)^{1-\eta}} \operatorname{ctg} \pi(1-\eta)(b-a)^{1-\eta} \widetilde{\mathcal{F}}_{2}(b) \\
& =\frac{\left(\sqrt{1+\beta^{2}}+\beta\right)}{2 \sqrt{1+\beta^{2}}} F_{2}(b)+\frac{-\operatorname{ctg} \pi \eta}{2 \sqrt{1+\beta^{2}}} F_{2}(b)=0
\end{aligned}
$$

(here we have used the relation $\operatorname{ctg} \pi \eta=\left(\sqrt{1+\beta^{2}}+\beta\right)$ ). The proof of the lemma is complete.

10. Singularities of $\nabla u$ may disappear. It follows from the formulas of Theorem 5 , that if $\rho_{j}^{d}(d)=0$ for $j=1,2$, then $\nabla u(x)$ has no singularities in the point $x(d) \quad(d=a$ or $d=b)$, and vice versa. There are no singularities at both ends of $\Gamma$ if the following conditions hold:

$$
\rho_{j}^{a}(a)=0, \quad \rho_{j}^{b}(b)=0, \quad j=1,2 .
$$

Actually, (75) contains four requirements for the functions $f^{+}(s)$ and $f^{-}(s)$.

LEMma 9. (1) If $\rho_{1}^{d}(d)=\rho_{2}^{d}(d)=0$ where $d=a$ or $d=b$, then the solution gradient of Problem $\mathcal{S}$ is continuous at the end $x(d)$ of the cut $\Gamma$.

(2) If $\rho_{1}^{d}(d)=\rho_{2}^{d}(d)=0$ for $d=a$ and $d=b$, then the solution gradient of Problem $\mathcal{S}$ is continuous at both ends of the cut $\Gamma$.

We study the following question: under what conditions on the functions $f^{+}(s)$ and $f^{-}(s)$ from (3) and (4), do the singularities of the solution gradient of Problem $\mathcal{S}$ at the ends of $\Gamma$ disappear? The analytic investigation for a cut of an arbitrary shape is quite difficult. We explore a certain particular case. Namely, let $\Gamma$ be a segment: $\Gamma=\{x: x(s)=(s \cos \theta, s \sin \theta), s \in[a, b]\}$. Then $Y_{1}(s, \sigma)=Y_{2}(s, \sigma) \equiv 0$ for $s, \sigma \in \Gamma$, and Eqs. (45) - (49) have solutions

$$
\begin{aligned}
& \rho_{1}(s)=\frac{\left(\sqrt{1+\beta^{2}}-\beta\right)}{2 \sqrt{1+\beta^{2}}} F_{1}(s)+\frac{1}{2 \pi \sqrt{1+\beta^{2}} Q_{1}(s)} \int_{\Gamma} \frac{F_{1}(\xi) Q_{1}(\xi)}{\xi-s} d \xi-\frac{B_{1} \cos \pi \eta}{Q_{1}(s)} \\
& \rho_{2}(s)=\frac{\left(\sqrt{1+\beta^{2}}+\beta\right)}{2 \sqrt{1+\beta^{2}}} F_{2}(s)-\frac{1}{2 \pi \sqrt{1+\beta^{2}} Q_{2}(s)} \int_{\Gamma} \frac{F_{2}(\xi) Q_{2}(\xi)}{\xi-s} d \xi-\frac{B_{2} \sin \pi \eta}{Q_{2}(s)}
\end{aligned}
$$


where $s \in \Gamma$,

$$
\begin{gathered}
Q_{1}(\xi)=(\xi-a)^{1 / 2-\eta}(b-\xi)^{1 / 2+\eta}, \quad Q_{2}(\xi)=(\xi-a)^{1-\eta}(b-\xi)^{\eta}, \quad \xi \in \Gamma, \\
F_{1}(s)=2\left(\sqrt{1+\beta^{2}} f^{\prime}+(s)+f^{-}(s)\right), F_{2}(s)=2\left(\sqrt{1+\beta^{2}}-\beta\right)\left(\sqrt{1+\beta^{2}} f^{\prime}+(s)-f^{-}(s)\right),
\end{gathered}
$$

the constants $B_{1}, B_{2}$ are given by (52). We compute the functions $\rho_{j}^{d}(s)$ using (61) and (62):

$$
\begin{gathered}
\rho_{1}^{a}(a)=\frac{1}{2 \pi \sqrt{1+\beta^{2}}(b-a)^{1 / 2+\eta}} \int_{a}^{b} F_{1}(\xi)\left(\frac{b-\xi}{\xi-a}\right)^{1 / 2+\eta} d \xi-\frac{B_{1} \cos \pi \eta}{(b-a)^{1 / 2+\eta}}, \\
\rho_{1}^{b}(b)=-\frac{1}{2 \pi \sqrt{1+\beta^{2}}(b-a)^{1 / 2-\eta}} \int_{a}^{b} F_{1}(\xi)\left(\frac{\xi-a}{b-\xi}\right)^{1 / 2-\eta} d \xi-\frac{B_{1} \cos \pi \eta}{(b-a)^{1 / 2-\eta}}, \\
\rho_{2}^{a}(a)=-\frac{1}{2 \pi \sqrt{1+\beta^{2}}(b-a)^{\eta}} \int_{a}^{b} F_{2}(\xi)\left(\frac{b-\xi}{\xi-a}\right)^{\eta} d \xi-\frac{B_{2} \sin \pi \eta}{(b-a)^{\eta}}, \\
\rho_{2}^{b}(b)=\frac{1}{2 \pi \sqrt{1+\beta^{2}}(b-a)^{1-\eta}} \int_{a}^{b} F_{2}(\xi)\left(\frac{\xi-a}{b-\xi}\right)^{1-\eta} d \xi-\frac{B_{2} \sin \pi \eta}{(b-a)^{1-\eta}} .
\end{gathered}
$$

We are going to satisfy all the conditions (75) at once, so that $\nabla u(x)$ will be continuous at the points $x(a)$ and $x(b)$. We seek the functions $F_{1}(\xi), F_{2}(\xi)$ in the form

$$
\begin{gathered}
F_{1}(\xi)=(\xi-a)^{1 / 2+\eta}(b-\xi)^{1 / 2-\eta} G_{1}(\xi) \in C^{0, \lambda}(\Gamma), \\
F_{2}(\xi)=(\xi-a)^{\eta}(b-\xi)^{1-\eta} G_{2}(\xi) \in C^{0, \lambda}(\Gamma), \quad \lambda \in(0,1] .
\end{gathered}
$$

Then conditions (75) for $F_{1}(\xi)$ and $F_{2}(\xi)$ become the following conditions for the functions $G_{1}(\xi), G_{2}(\xi)$ :

$$
\int_{a}^{b} G_{j}(\xi)(\xi-a) d \xi=A l_{j}, \quad \int_{a}^{b} G_{j}(\xi)(b-\xi) d \xi=-A l_{j}, \quad j=1,2,
$$

or

$$
\int_{a}^{b} G_{j}(\xi) d \xi=0, \quad \int_{a}^{b} \xi G_{j}(\xi) d \xi=A l_{j}, \quad j=1,2,
$$

where $l_{1}=8 \pi\left(1+\beta^{2}\right) \sin \pi \eta \cos ^{2} \pi \eta, l_{2}=8 \pi\left(1+\beta^{2}\right) \sin ^{3} \pi \eta$.

Note (see (76) ) that to satisfy the condition $F_{1}(s), F_{2}(s) \in C^{0, \lambda}(\Gamma)$ with some (i.e., not given before) $\lambda \in(0,1]$, it suffices that $G_{1}(s), G_{2}(s) \in C_{\eta_{0}}^{\omega_{0}}(\Gamma)$ with an arbitrary $\omega_{0} \in(0,1]$ and $\eta_{0}=\min \{\eta, 1 / 2-\eta\}$.

Lemma 10. Let $\Gamma=\{x: x=x(s)=(s \cos \theta, s \sin \theta), s \in[a, b]\}$, and let $F_{1}(s), F_{2}(s)$ be defined in (76), and $G_{1}(s), G_{2}(s)$ satisfy Eq. (77). Then the solution gradient of Problem $\mathcal{S}$ is continuous at the ends of the cut $\Gamma$. 
We give several ways to construct the functions $G_{1}(s), G_{2}(s)$. Below we assume that the segment $[a, b]$ is simplified by a linear change of variables.

(I) We put $a=-b<0$.

(1) Let $G_{1}(s), G_{2}(s)$ be even functions, and let $A=0$. Then to satisfy the homogeneous conditions (77), it suffices to require that $\int_{0}^{b} G_{j}(\xi) d \xi=0$ for $j=1,2$. For instance, we can choose $G_{1}(\xi)=G_{2}(\xi)=C(b-2|\xi|)$, where $C$ is an arbitrary constant.

(2) Let $G_{1}(s), G_{2}(s)$ be odd functions. If $A=0$, then to satisfy (77), it suffices to require that $\int_{0}^{b} \xi G_{j}(\xi) d \xi=0$ for $j=1,2$. In the general case, when $A$ is an arbitrary constant, it suffices to require that

$$
\int_{0}^{b} \xi\left(G_{j}(\xi)-\frac{(2 k+1) l_{j} A}{2 b^{2 k+1}} \xi^{2 k-1}\right) d \xi=0,
$$

where $j=1,2$, and $k$ is a natural number. In particular, if $A \neq 0$, we can choose

$$
G_{j}(s)=(2 k+1) 2^{-1} l_{j} A \xi^{2 k-1} / b^{2 k+1}, \quad j=1,2 .
$$

We can proceed in a similar way if one of the functions $G_{1}(s), G_{2}(s)$ is odd and the other is even.

(II) Below we assume $a=-1, b=1$. In this case, it is convenient to rewrite requirement (77) in terms of Legendre polynomials [11, 12. We recall that Legendre polynomials $\mathcal{P}_{n}(s), n=0,1, \ldots$, generate a closed orthogonal system in the space $L_{2}(-1,1)$. Besides, $\left\|\mathcal{P}_{n}\right\|^{2}=2 /(2 n+1), \mathcal{P}_{0}(s)=1, \mathcal{P}_{1}(s)=s$. Putting

$$
G_{j}(s)=\sum_{n=0}^{\infty} \mathcal{A}_{n}^{j} \mathcal{P}_{n}(s), \quad j=1,2,
$$

and assuming that the series converges in the mean, we define from (77): $\mathcal{A}_{0}^{j}=0$, $\mathcal{A}_{1}^{j}=3 A l_{j} / 2, j=1,2$. Other coefficients $\mathcal{A}_{n}^{j}$ remain undefined $(j=1,2, \quad n=2,3, \ldots)$.

Thus, let $\mathcal{A}_{n}^{j}$ be arbitrary coefficients $(j=1,2, \quad n=2,3, \ldots)$ such that the series $\sum_{n=2}^{\infty} \mathcal{A}_{n}^{1} \mathcal{P}_{n}(s)$ and $\sum_{n=2}^{\infty} \mathcal{A}_{n}^{2} \mathcal{P}_{n}(s)$ converge in the mean. Then functions

$$
G_{j}(s)=\frac{3 A l_{j}}{2} s+\sum_{n=2}^{\infty} \mathcal{A}_{n}^{j} \mathcal{P}_{n}(s), \quad j=1,2,
$$

satisfy the conditions (77).

The smoothness requirements for $G_{1}(s)$ and $G_{2}(s)$ in (76) admit that $G_{1}(s)$ and $G_{2}(s)$ may have integrable power-law singularities in the points $s= \pm 1$. To simplify the argumentation, we consider the convergence of the series in (78) to Hölder functions.

Using the Weierstrass sufficient test for uniform convergence and the fact that $\mathcal{P}_{n}(s)$ are uniformly bounded on the segment $[-1,1]$ [12, Chapter 4, Sec. 2], one can prove the sufficient condition of uniform convergence of the series in (78):

Lemma 11. Let $\mathcal{A}_{n}^{j}=O\left(1 / n^{2+\epsilon}\right)$ as $n \rightarrow \infty$, where $\epsilon>0, j=1,2$. Then the series in (178) converge uniformly, and the functions $G_{1}(s)$ and $G_{2}(s)$ belong to the class $C^{1}(\Gamma)$ and satisfy conditions (77).

The result of item (II) is the following. 
THEOREM 6. Let

(1) $\Gamma=\{x: x=x(s)=(s \cos \theta, s \sin \theta), s \in[-1,1]\}$;

(2) the functions $F_{1}(s), F_{2}(s)$ be defined by (76), (78);

(3) $\mathcal{A}_{n}^{j}$ be arbitrary coefficients $(n=2,3, \ldots)$, such that the series in (78) converge in the mean to Hölder functions for $j=1$ and $j=2$.

Then the solution gradient of Problem $\mathcal{S}$ is continuous at the ends of the cut $\Gamma$.

The work was partly supported by the RFBR grants no. 05-01-00050, 06-01-00001.

\section{REFERENCES}

[1] P.A. Krutitskii, A.I. Sgibnev. The method of integral equations in the mixed Dirichlet-Neumann problem for the Laplace equation in the exterior of cuts in a plane. Differential Equations. 2001. Vol. 37. No 10. pp. 1363-1375. MR.1945240 (2003g:35037)

[2] A.S. Gosteva, N.Ch. Krutitskaya, P.A. Krutitskii. Mixed problem in a magnetized semiconductor film with cuts along a straight line. Fundamentalnaya i Prikladnaya Matematika 2000. Vol. 6. No 4. pp. 1061-1073. MR.1813011 (2001k:78016)

[3] S.A. Gabov. The angular potential and some of its applications. Math. U.S.S.R. Sbornik. 1977. Vol. 32, pp. 423-436. MR0463457 (57:3407)

[4] P.A. Krutitskii. The Dirichlet problem for the Helmholtz equation in the exterior of cuts in the plane. Comp. Math. Math. Phys. 1994. Vol. 34. No 8-9. pp. 1073-1090. MR1300397 (95f:35046)

[5] P.A. Krutitskii. The Neumann problem for the Helmholtz equation in the exterior of cuts in the plane. Comp. Math. Math. Phys. 1994. Vol. 34. No 11. pp. 1421-1431. MR.1307611 (95i:45004)

[6] N.I. Muskhelishvili. Singular integral equations. Noordhoff, Groningen, 1972; 3rd Russian ed.: Nauka Publishers, Moscow, 1968.

[7] A.N. Kolmogorov, S.V. Fomin. Elements of the Theory of Functions and Functional Analysis. Dover, New York, 1999.

[8] S.G. Krein (editor). Functional analysis. Nauka Publishers, Moscow, 1964.

[9] P.A. Krutitskii. On the electric current from electrodes in a magnetized semiconductor film. IMA J. of Appl. Math., 1998, v. 60, p. 285-297.

[10] P.A. Krutitskii. A mixed problem for the Laplace equation outside a system of cuts in a plane. Differential Equations. 1997. Vol. 33. No 9. pp. 1184-1193. MR.1638915 (99h:35030)

[11] A.F. Nikiforov, V.B. Uvarov. Special Functions of Mathematical Physics: A Unified Introduction With Applications. Birkhäuser, Basel, 1988. MR0922041 (89h:33001)

[12] P.K. Suetin. Classical Orthogonal Polynomials. Nauka Publishers, Moscow, 1979. MR0548727 (80h:33001) 